\title{
Analysis of Individual Compact Fission Product Inventory and Burnup for the AGR-1 TRISO Experiment using Gamma Spectrometry
}

\author{
Jason M. Harp
}

December 2010

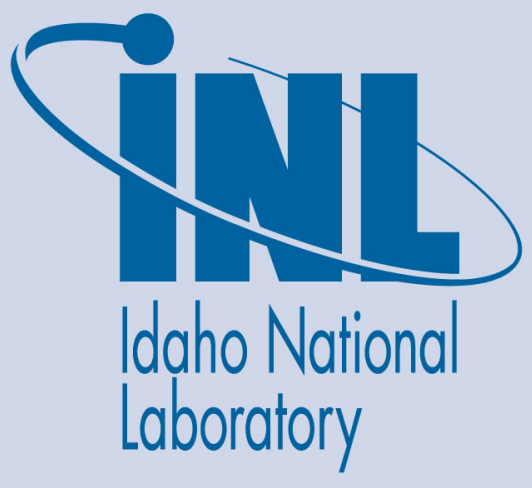

The INL is a U.S. Department of Energy National Laboratory operated by Battelle Energy Alliance 


\title{
Analysis of Individual Compact Fission Product Inventory and Burnup for the AGR-1 TRISO Experiment using Gamma Spectrometry
}

\author{
Jason M. Harp
}

December 2010

Idaho National Laboratory Idaho Falls, Idaho 83415

http://www.inl.gov

Prepared for the

U.S. Department of Energy

Under DOE Idaho Operations Office

Contract DE-AC07-05ID14517 
Rev. 4

Analysis of Individual Compact Fission Product Inventory and Burnup for the AGR-1 TRISO Experiment using Title: Gamma Spectrometry

1. Index Codes
Building/Type: N/A
SSC ID: N/A
Site Area: N/A

2. Quality Level and Determination No.:

\section{QL-2, MFC-001009}

3. Objective/Purpose:

The objective of this document is to describe a method for non-destructive burnup evaluation of the AGR-1 TRISO fuel compacts using gamma spectrometry. Initially, gamma-ray spectra taken from the AGR-1 experiment TRISO fuel compacts using the Precision Gamma Scanner (PGS) system were analyzed. The spectrometry analysis included a characterization of the PGS system and the measured fission product activities for the compacts. The measured activities were compared to detailed production/depletion fuel inventory simulations of the AGR-1 experiment. Two methods for relating burnup to measured fission product activity were developed from the results of the fuel inventory simulations and applied to the measured fission product activities. Burnup derived from the PGS measurements was compared to the predicted burnup from simulation.

4. Conclusions/Recommendations:

The collected PGS spectra were successfully analyzed to find the measured fission product inventory of each AGR-1 TRISO fuel compact. The measured fission product activities compared favorably to the predicted fission product fuel compact activities in each capsule for several different fission products. The numerical results of this analysis are included in this document. Burnup values derived from the results of the gamma spectrometry analysis were also compared to predicted burnup values. The measured and predicted values also compared favorably and are presented in this document for all 72 AGR-1 compacts.

5. Review (R) and Approval (A) and Acceptance (Ac) ${ }^{1}$ :

\begin{tabular}{|c|c|c|c|}
\hline & & Typed Name/Organization & Signature or eCR No. ${ }^{2}$ \\
\hline Performer/Author & & Jason M. Harp / C310 & eCR 623131 \\
\hline Technical Checker & $\mathrm{R}$ & Heather Chichester/ C620 & eCR 623131 \\
\hline Independent Peer Reviewer ${ }^{3}$ & $\mathrm{R}$ & $\mathrm{N} / \mathrm{A}$ & \\
\hline Performer's Manager & A & T.A. Hyde / C310 & eCR 623131 \\
\hline Requester & Ac & Paul A. Demkowicz / C620 & eCR 623131 \\
\hline Quality Assurance & Ac & Kirk W. Bailey / Q510 & eCR 623131 \\
\hline \multicolumn{4}{|c|}{1 Review and Approval are required. See LWP-10200 for definitions and responsibilities. } \\
\hline \multicolumn{4}{|c|}{$\begin{array}{l}2 \text { Electronic Change Request (eCR) numbers in lieu of signatures on this page indicate electronic final review, approval and acceptance by } \\
\text { the listed individuals. }\end{array}$} \\
\hline \multicolumn{4}{|c|}{3 If required, per LWP-10200. } \\
\hline \multicolumn{4}{|c|}{$4 \quad$ Required if the ECAR contains safety software validation. } \\
\hline
\end{tabular}




\section{CONTENTS}

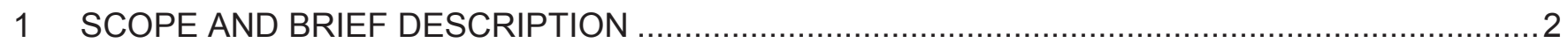

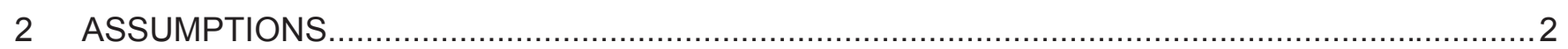

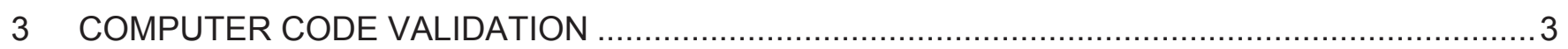

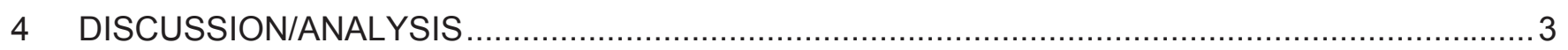

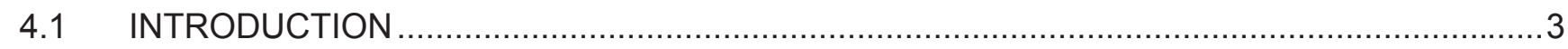

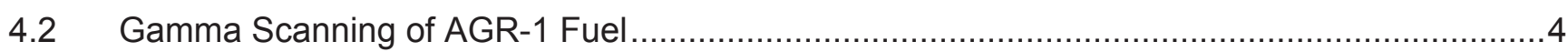

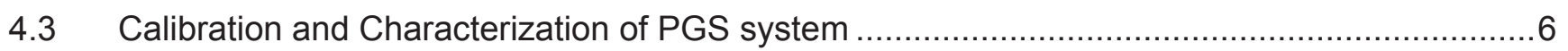

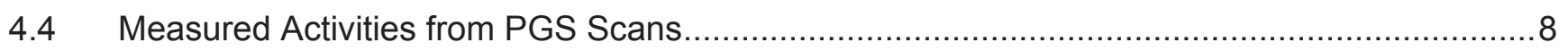

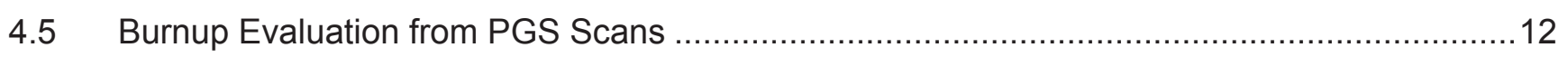

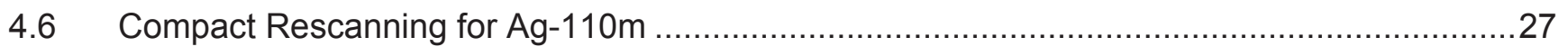

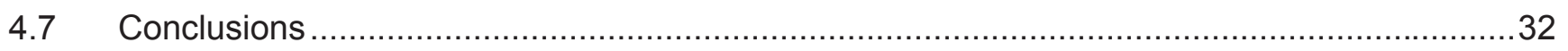

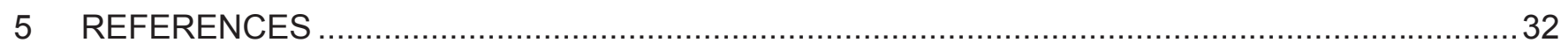

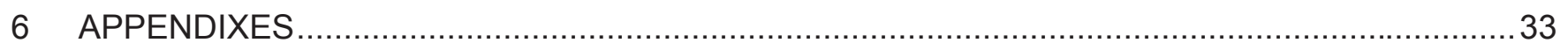

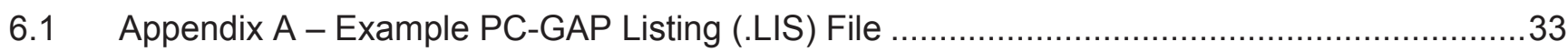

6.2 Appendix B - Listing File Activity Stripping Script and Output ......................................39

\section{SCOPE AND BRIEF DESCRIPTION}

Gamma-ray scans of fuel compacts from the AGR-1 (Advanced Gas Reactor-1) TRISO fuel irradiation test have been collected using the Precision Gamma Scanner (PGS) system located in the Hot Fuel Examination Facility (HFEF) of the Materials and Fuels Complex (MFC) at Idaho National Laboratory (INL). The AGR-1 test contained 72 TRISO fuel compacts that were irradiated in the INL Advanced Test Reactor (ATR) to a burnup of 11.5 to $19.6 \%$ FIMA (fissions per initial heavy-metal atom; estimate from simulation).1 This document will detail the configuration of the PGS system during these measurements along with the calibration of the PGS system for gamma-ray spectrum collection. The PGS system collects spectral data from an ORTEC MCB (Multi-Channel Buffer) using ORTEC software including Connections32 and Maestro. Spectral data from the PGS is saved as a uniquely named ORTEC binary spectrum file (CHN). Additional data detailing the position and state of the PGS system is also stored as an identically named dat file. PGS spectral data from the PGS system was analyzed using the PC-GAP gamma-ray spectrum analysis package developed at INL. The parameters of this analysis are detailed in this document. After spectral analysis, the activities of the isotopes of interest were collected and organized into a usable format using a python script, which will be included as an appendix to this document. The fission product inventory of each compact will be used to non-destructively evaluate the burnup of each fuel compact. Burnup evaluation utilizing gamma-ray spectrometry is a well established non-destructive technique for examining nuclear fuel performance following irradiation. $2^{3}$ The measured burn-up from the PGS scans will then be compared to detailed computer simulations of the AGR-1 experiment.

\section{ASSUMPTIONS}

Spectra received from the PGS system were examined to assure there were no obvious problems with the collected data. All activity and burnup calculations assume the spectra used in their derivation were collected under the same PGS conditions. Calibration sources were scanned in the PGS scanning stack before and after each set of fuel compacts to help assure that there were no major deviations in the high purity germanium (HPGe) detector conditions over the course of a 
Analysis of Individual Compact Fission Product Inventory and Burnup for the AGR-1 TRISO Experiment using Title: Gamma Spectrometry

measurement. No major deviations were seen in the detector behavior based on calibration source behavior in the final measurements.

\section{COMPUTER CODE VALIDATION}

Code 1:
a. Computer type: PC running Windows XP
b. Computer program name and revision: Maestro for Windows Version 6.08,
c. Inputs: PGS System
d. Outputs: See PGS formal data transmittal of collected spectra
e. Evidence of, or reference to, computer program validation: The data was generated by the PGS system, owned and managed by HFEF under their own software management controls. No software management to Maestro is documented in this ECAR.

Code 2:
a. Computer type: PC running Windows XP
b. Computer program name and revision: PC-GAP (Convert module) Version 01/16/2008
c. Inputs: See PGS formal data transmittal of collected spectra
d. Outputs: See Sample in Appendix A
e. Evidence of, or reference to, computer program validation: PCGAP is "legacy" software per LWP-13620, so, although software QA documentation for it is not complete, it has been accepted and is used under the terms of legacy software in LWP-13620. It is managed as documented in Lab Notebook LAB-764.

\section{Code 3:}

a. Computer type: PC running Windows XP

b. Computer program name and revision: Python 2.7

c. Inputs Mult_LIS_acts.py (see Appendix B)

d. Outputs: See Sample in Appendix B

e. Evidence of, or reference to, computer program validation: A Python script extracts select data from the PCGAP file, and prepares it for use in a spreadsheet for graphing purposes. Neither the Python script nor the spreadsheet changes the data values. The visual presentation of the data in the resulting charts provides a verification that PCGAP and the Python script are functioning correctly.

\section{DISCUSSION/ANALYSIS}

\subsection{INTRODUCTION}

Gamma-ray scans of the AGR-1 (Advanced Gas Reactor-1) TRISO fuel irradiation experiment have been analyzed to non-destructively evaluate the burnup of individual fuel compacts. Burnup evaluation utilizing gamma-ray spectrometry is a well established non-destructive technique for examining nuclear fuel performance following irradiation. $2^{3}$ In this work, the fuel compact burnup from gamma-ray spectrometry will be compared to detailed computer simulations of the AGR-1 experiment.

The AGR-1 experiment is part of a series of low-enriched uranium oxycarbide TRISO coated particle fuel experiments that are ongoing at several research facilities of the Idaho National Laboratory (INL) and Oak Ridge National Laboratory. The experiment contained six temperature-controlled irradiation capsules that each held 12 cylindrical TRISO fuel compacts ( 1 inch long x 0.5 inch diameter) consisting of approximately 4100 individual TRISO particles per compact. 1 AGR-1 was irradiated for 620 Effective Full Power Days in the Advanced Test Reactor (ATR) located at INL, and is estimated to have reached a peak burnup of 19.5\% FIMA (Fissions of Initial Metal Atoms). 4 After irradiation the experiment was sent to the Materials and Fuels Complex (MFC) at INL for post-irradiation examination.

Burnup is typically derived from gamma-ray spectrometry data using two methods: (1) absolute activity evaluation of direct fission products and (2) activity ratio of a shielded fission product and a direct fission product. Direct fission products are defined as being produced directly from heavy metal fission as the original fission fragment or by the beta decay of the original fission fragments. Shielded fission products are created when a neutron capture reaction takes place in a previously produced direct fission product and the newly produced isotope is shielded from direct fission product production through a beta minus decay chain by a stable nuclide. An example of a direct fission product is Cs-137, and an example of a shielded 
Analysis of Individual Compact Fission Product Inventory and Burnup for the AGR-1 TRISO Experiment using Title: Gamma Spectrometry

fission product is Cs-134. For an isotope to be useful as a burnup indicator it should have a half-life that allows it to monotonically increase in activity as a function of burnup. This requires an isotope to have a half-life on the order or longer than the time period of the irradiation. Additionally the indicator must not decay below detectable limits between the end of irradiation and measurement on a spectrometry system. For the AGR-1 experiment, Cs-137, Sb-125, Cs-134, and Eu-154 are potential burnup indicators for both the direct and ratio methods.

\subsection{Gamma Scanning of AGR-1 Fuel}

One component of the AGR-1 post irradiation examination was the gamma-scanning of all 72 fuel compacts using the Precision Gamma Scanner (PGS) system. The PGS system consists of a collimator that penetrates the HFEF hot cell wall, an HPGe gamma spectrometry system, and a set of in-cell devices used to position samples for examination. The hot cell side of the collimator consists of tungsten alloy blocks that can be adjusted to create a slit height varying from 0.1 inches to 0.001 inches. The width of the collimator opening is fixed at 0.875 inches creating a rectangular viewing area for the PGS system. Gamma-rays entering the collimator travel through the hot cell wall, through a second tungsten alloy beam scraper, and then to the HPGe detector. An assembly drawing of the PGS collimator is shown in Fig. 1.

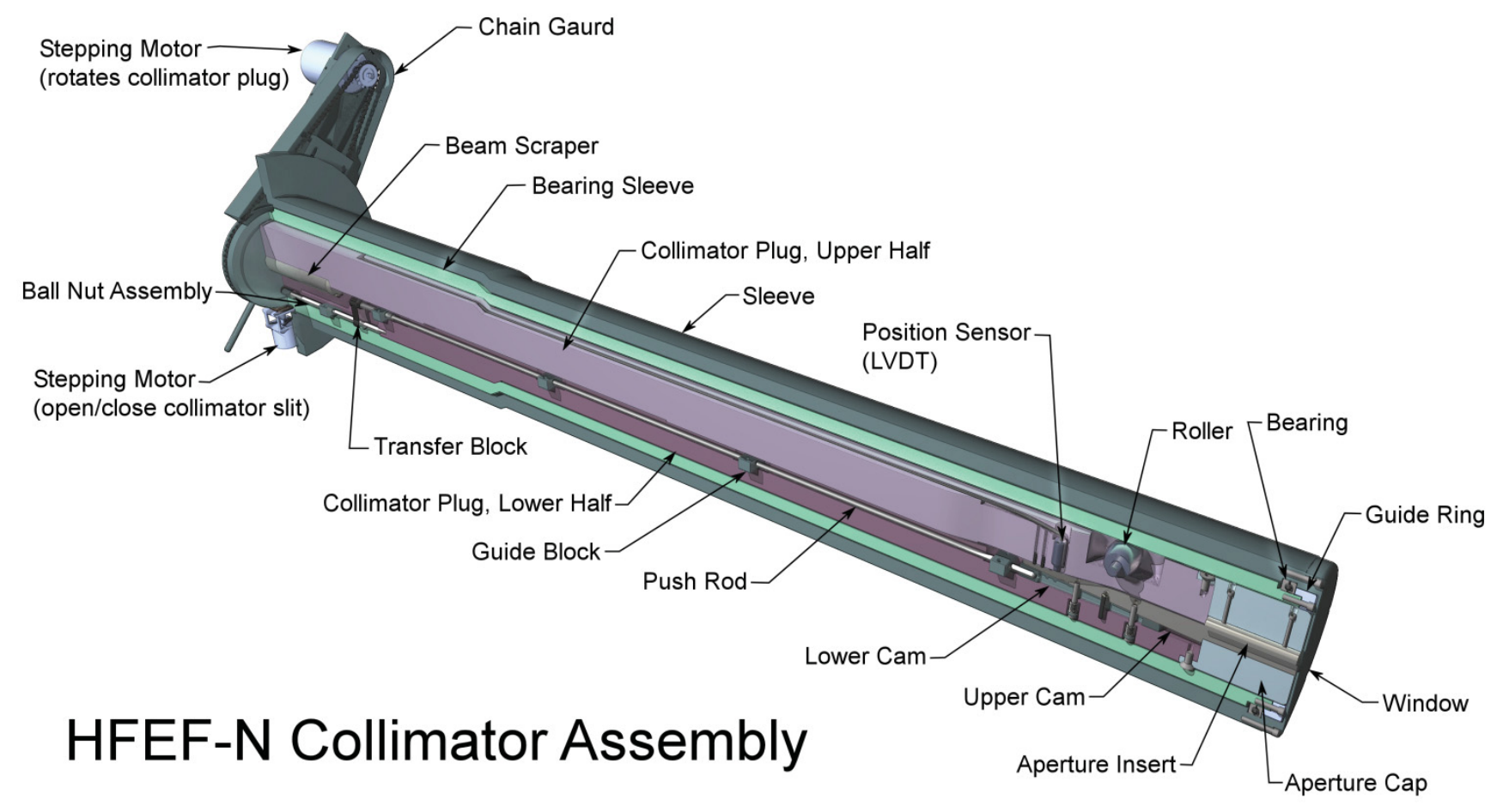

Fig. 1. Assembly drawing of PGS collimator5

The PGS uses a custom control software interface that controls both the collection of gamma-ray spectra and the movement of the sample in the hot cell. Spectroscopic control is achieved by communicating with an ORTEC 921 MultiChannel Buffer (MCB). Once the sample is positioned the PGS collects a spectrum until the live time clock on the MCB reaches the desired limit. The contents of the buffer are then stored on the PGS control computer as a uniquely named ORTEC .CHN binary spectrum file. Additionally a corresponding .DAT file that contains position and system status information related to the PGS during spectrum collection is also saved after each spectrum is collected. All the .CHN and .DAT files collected in scans of the AGR-1 compacts have been archived as an official data transmittal in the NGNP project files.6 The control software then moves the sample to the next desired position and begins counting again.

For the AGR-1 PGS examination, each compact was loaded into an individual aluminum container.7 The containers containing the fuel compacts were then stacked axially in an additional aluminum tube8 that was connected to the PGS and progressively scanned in 0.1 inch intervals with the collimator slit set to 0.1 inches. The slit was oriented perpendicular to 
the compact axis such that each scan captured a 0.1 inch axial "slice" of the compact. A single scan contained all 12 fuel compacts from one of the six AGR-1 capsules. Additionally, preceding and following the containers containing the fuel compacts were well characterized Eu-152 calibration sources9 used to diagnose any potential shifts in detection system behavior during a scan. The container preceding the fuel compacts contained a single Eu- 152 source, ${ }^{10}$ and the container following the fuel compacts contained two Eu-152 sources. ${ }^{11}$ The loading order of compacts was the same for all capsules as shown in Table 1. An illustration of the numbering system used to identify individual compacts can be found in Fig. 2 and further details can be found in Reference 12. Each set of compacts was scanned at separate dates. The start date of scanning for the compacts of each capsule is shown in Table 2.

Before any scans were completed, the PGS stack was centered on the PGS collimator by turning the collimator to its vertical position and scanning across Eu-152 calibration sources to determine the edges of the Eu-152 source. The midpoint of the two edges where the source was detectable was then determined to be the center of the PGS stack. Given the design of the PGS system, misalignment of the collimator with the PGS stack could only result in a very small loss of data from the AGR-1 compacts. The geometric alignment for the worst case scenario was analyzed. For this case, the un-scanned portion of the compact is only about 1.1 to $1.4 \%$ of the total volume. This possible missing volume is small compared to the uncertainty on the activity of most measured isotopes which is usually greater than $1 \%$ from counting statistics alone not including other sources of uncertainty.

Table 1. Scan order for all compacts

\begin{tabular}{|c|c|}
\hline Order & Compact ID \\
\hline \multirow[t]{13}{*}{ Top } & Source - G6274 \\
\hline & AGR-1 $* 43$ \\
\hline & AGR-1 *33 \\
\hline & AGR-1 $* 23$ \\
\hline & AGR-1 *13 \\
\hline & AGR-1 $* 42$ \\
\hline & AGR-1 $* 32$ \\
\hline & AGR-1 $* 22$ \\
\hline & AGR-1 $* 12$ \\
\hline & AGR-1 $* 41$ \\
\hline & AGR-1 $* 31$ \\
\hline & AGR-1 $* 21$ \\
\hline & AGR-1*11 \\
\hline Bottom & $\begin{array}{c}\text { Source - G6272 \& } \\
\text { G6273 }\end{array}$ \\
\hline
\end{tabular}

The * indicates any Capsule number. The first number after the * indicates the level of the compact in its capsule. The second number after the * indicates the stack the compact was in during irradiation. 


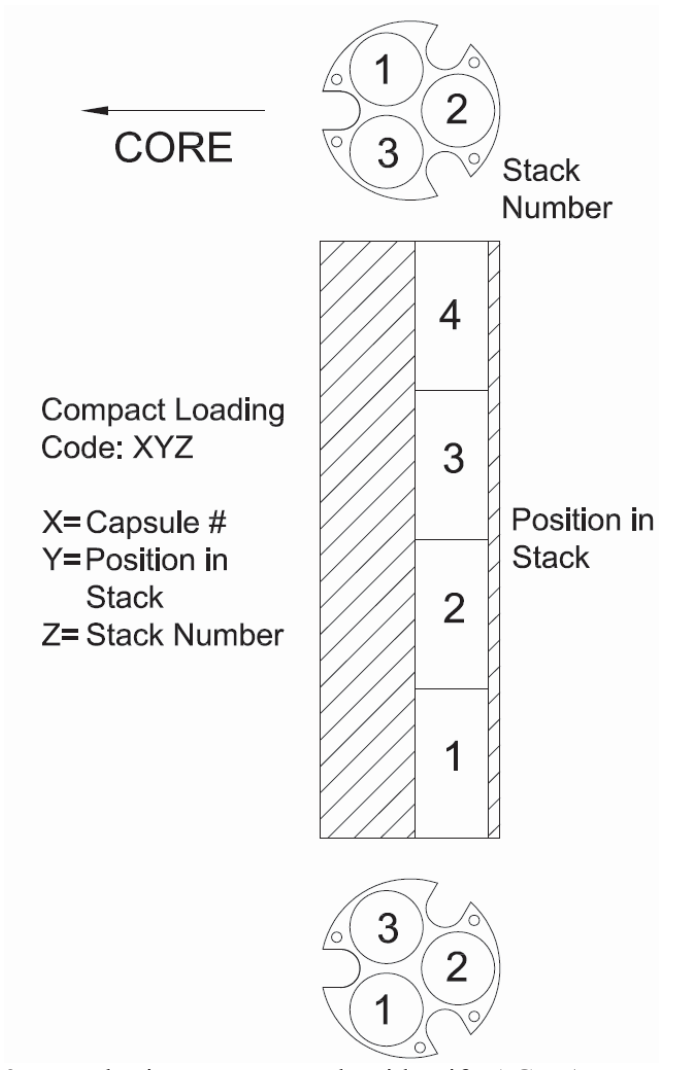

Fig. 2. Numbering system used to identify AGR-1 compacts.

Table 2. Collection start date of spectra for compacts from different capsules

\begin{tabular}{|c|c|}
\hline Capsule & Date \\
\hline 1 & $6 / 17 / 2010$ \\
\hline 2 & $1 / 5 / 2011$ \\
\hline 3 & $8 / 16 / 2010$ \\
\hline 4 & $6 / 3 / 2010$ \\
\hline 5 & $8 / 11 / 2010$ \\
\hline 6 & $6 / 11 / 2010$ \\
\hline
\end{tabular}

\subsection{Calibration and Characterization of PGS system}

Gamma-ray spectra for each incremental scan were analyzed to determine the activity of various different fission products present in the fuel compacts. The collected gamma-ray spectra were analyzed using the PC-GAP software package developed at INL. In order to use PC-GAP to convert counts in the collected spectra to activity present in the fuel compacts, an energy calibration and an efficiency calibration for the TRISO fuel compacts needed to be derived.

The energy calibration was performed and confirmed by using the spectra collected from the three Eu- 152 sources as they were scanned during each PGS scan of a particular capsule. Comparison of peak channel positions from the top Eu-152 source and the bottom two Eu-152 sources confirmed that the PGS detector system was stable throughout the entire PGS scan of the complete fuel compact stack which consisted of about 420 scans per capsule (approximately 75 to 80 hours with dead time in the detector).

The creation of an efficiency calibration for the AGR-1 compacts was complicated by the lack of a calibrated standard that could faithfully replicate the geometry present in a fuel compact. In order to account for the geometry effects of the 
Analysis of Individual Compact Fission Product Inventory and Burnup for the AGR-1 TRISO Experiment using Title: Gamma Spectrometry

TRISO fuel compact, a Monte Carlo N-Particle (MCNP) model was created of the PGS collimator, the PGS detector, the Eu152 calibration source geometry, and a representative AGR-1 fuel compact. Initially the simulated geometry represented the case of a Eu-152 source being viewed by the PGS collimator. The MCNP model was refined until efficiency curves derived from the simulation closely matched measured efficiency curves from the in cell Eu-152 sources. The MCNP source geometry was then changed to the AGR-1 geometry. The AGR-1 compact model included particles with the exact geometry of the AGR-1 particles with all their corresponding layers. ${ }^{13}$ In the model, spheres of coated particles were surrounded by a cube of graphite large enough to replicate the AGR-1 packing fraction of 35\%. An MCNP URAN card was applied to the coated sphere so that it was randomly located in the surrounding graphite. Source particles were initiated inside kernels of the coated spheres to represent fission products trapped in the fuel. The MCNP source was further restricted to only start particles in the slice of compact that was directly viewed by the PGS collimator. This was done to create an efficiency curve for the slice of fuel compact that was being viewed by the collimator, not an efficiency of the entire compact. Additional simulations were run of the slice above and below the viewed slice so that their contribution $(\sim 1 \%)$ to the viewed slice efficiency could be considered. The slight differences between the Eu-152 source geometry and the fuel compact slice geometry were captured by the MCNP simulations and were used with the measured Eu-152 source efficiency curves to generate an efficiency curve for gamma-rays originating in the fuel compacts and being detected by the PGS detector system. These two efficiency curves were not widely different, and can be seen in Fig. 3. The major change occurs for gammas below $300 \mathrm{keV}$ where the TRISO fuel kernel and the layers of the TRISO fuel can cause some additional attenuation that is different from the attenuation seen by the Eu-152 source. These differences slightly change the efficiency curve magnitude and shape. The efficiency curve derived from simulation was then used in PC-GAP to process the collected spectra for activity per slice.

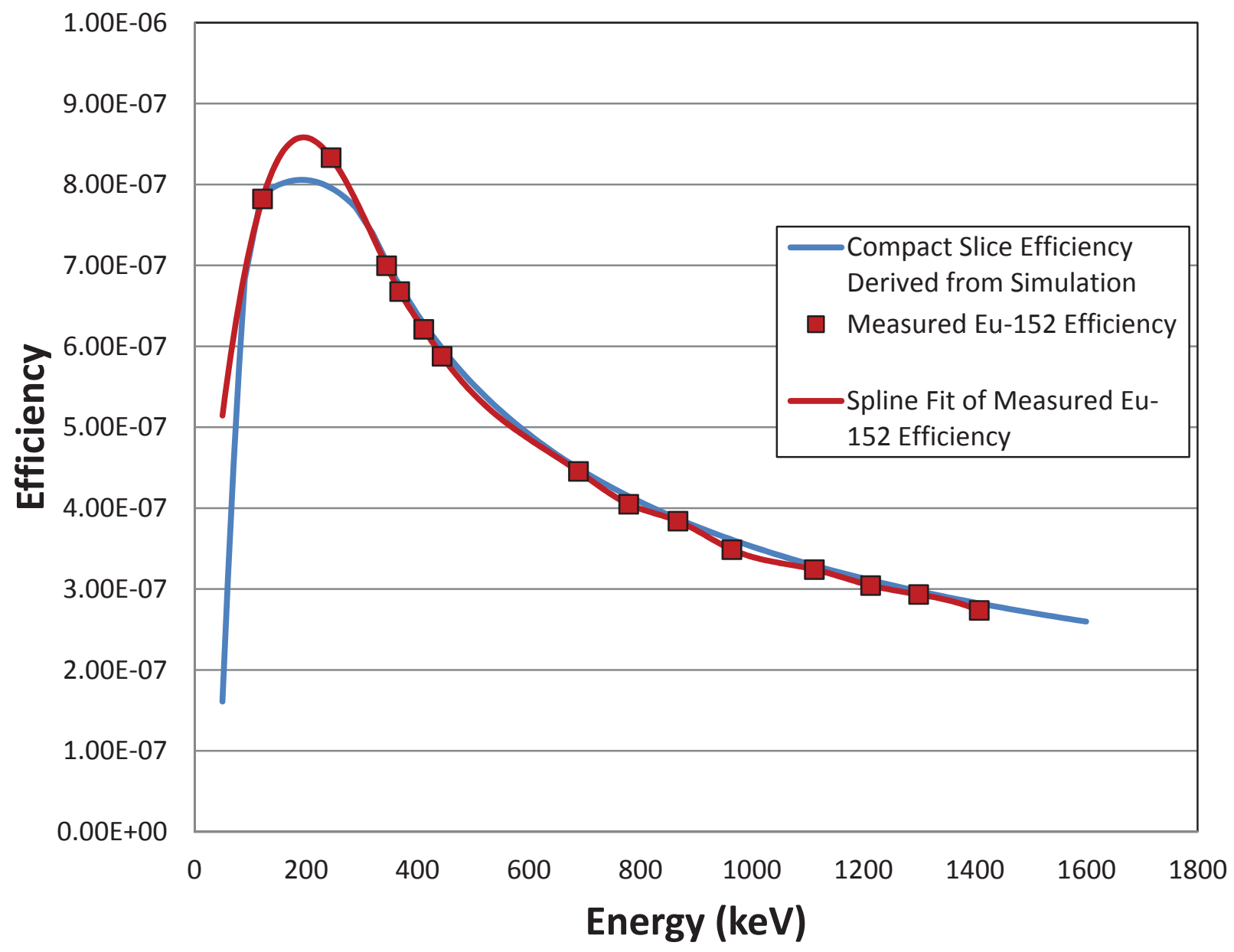

Fig. 3. Measured efficiency of Eu-152 source and derived efficiency of a slice of a compact. 
Analysis of Individual Compact Fission Product Inventory and Burnup for the AGR-1 TRISO Experiment using Title: Gamma Spectrometry

Each AGR-1 Capsule scan consisted of 421, 10 minute live time scans of a single 0.1 inch slice of the PGS scanning stack. $6^{8}$ The spectra were collected using and ORTEC 921 High-Rate Multichannel Buffer and saved as ORTEC .CHN files. These original .CHN files have been archived as an official data transmittal.6 These .CHN files were converted to PC-GAP format and analyzed using the PC-GAP CONVERT.EXE utility using the current version 2 PC-GAP analysis libraries. This process produces .DGE files that are the native spectrum file of PC-GAP and .LIS files that are text files containing the analysis of the converted spectrum (i.e. peak areas, peak identification, and activities). Pulling all the desired data out of the hundreds of .LIS files would be tedious and prone to human error. A python script was written to pull all the desired data out of each .LIS file. This python script and an example.LIS file are included in Appendix A of this document. There were 11 prominent fission products present in the scans: Y-91, Zr-95, Ru-103, Rh-106 (the daughter of Ru-106), Sb-125, Cs-134, Cs137, Ce-141, Ce-144, Eu-154, and Pr-144 (daughter of Ce-144). Activity information was pulled out of the .LIS files for these fission products in two ways. The preferred source of activity is from the Isotope Summary Table located in the .LIS file. In this table, the activity of an isotope is determined using a weighted average derived from all available gamma-ray peaks of a particular isotope. An alternative activity calculation was used to help identify the signal from low activity isotopes or signal from peaks whose centroids were further away from the library value than is allowed by PC-GAP. In the alternative method if an activity was not identified for one of the 11 isotopes listed above in the isotope summary table, the python script would look for a peak identified by PC-GAP in a $\pm 2 \mathrm{keV}$ energy range around the nuclide's primary gammaray. This range is twice the standard PC-GAP range of $\pm 1 \mathrm{keV}$. If a peak was found in that range the python script would convert its peak area into an activity using the peaks efficiency and the yield of the gamma-ray. Activities derived from the alternative method must be treated carefully since the wider energy range increase the possibility that a peak will be spuriously identified being emitted from a fission product of interest when the peak is actually background. After collecting all the desired activities and uncertainties, the python script would then move on to the next .LIS file until all files specified by the user were analyzed. It would then output a summary table of all the collected activities and activity uncertainties.

\subsection{Measured Activities from PGS Scans}

All the collected PGS spectra were analyzed using the method described in the Calibration and Characterization Section. Total activities for each compact and each isotope of interest were collected by summing the "slice" activities derived from spectra that corresponded to a certain AGR-1 compact. Table 3 to Table 8 contain the decay corrected measured activities for the different compacts. The activities are decay corrected back to the end of irradiation (November 6, 2009) based on the starting date of a PGS scan for a certain capsule. The PGS scan start dates are shown in Table 2. For most isotopes $\left(\mathrm{T}_{1 / 2-}\right.$ $>225$ days), any error in decay correction introduced by assuming a single scan start time is small compared to other errors in the activity calculations. Only 9 of the 11 detected isotopes are included in this list. Yttrium-91 is excluded from this list because of its very low activity, and the measured Ce-144 activity from its own gamma is excluded in favor of the activity measured from its daughter Pr-144. Pr-144 is the daughter of the longer lived Ce-144 and is in secular equilibrium with Ce144. Cerium-144 has a low energy gamma-ray $(133.5 \mathrm{keV})$ that is difficult to detect and properly convert into an activity, but Pr-144 produces two easily detectable gamma-rays. The tables begin with the fission products that are most useful for burnup determination by gamma-ray spectrometry. These isotopes include the shielded fission products Cs-134 and Eu-154 and direct fission products such as Cs-137, Sb-125, Ce-144 (by Pr-144), and Ru-106 (by Rh-106). Rhodium-106 is the gamma-ray producing daughter of the long lived Ru-106 which produces no gamma-rays when it decays. Other detected direct fission products like $\mathrm{Zr}-95$, Ru-103, and Ce-141 have shorter half-lives that would have reached or began approaching an equilibrium value in the AGR-1 test but are included in the tables for completeness. 
Rev. 4

Analysis of Individual Compact Fission Product Inventory and Burnup for the AGR-1 TRISO Experiment using

Title: Gamma Spectrometry

ECAR No.: 1682

ECAR Rev. No.: 3

Project File No.: 23843

Date: $05 / 21 / 2014$

Table 3. Decay corrected measured activities for AGR-1 Capsule 6

\begin{tabular}{|c|c|c|c|c|c|c|c|c|c|}
\hline \multirow[b]{2}{*}{ Compact ID } & \multicolumn{9}{|c|}{ Activity (Ci) } \\
\hline & Cs-137 & Cs-134 & Sb-125 & Eu-154 & $\begin{array}{l}\text { Pr-144/ } \\
\text { Ce-144 }\end{array}$ & $\begin{array}{c}\text { Rh-106/ } \\
\text { Ru-106 }\end{array}$ & Zr-95 & Ru-103 & Ce-141 \\
\hline AGR-1 643 & $3.66 \mathrm{E}-01$ & $2.68 \mathrm{E}-01$ & $1.94 \mathrm{E}-02$ & 8.34E-03 & $4.96 \mathrm{E}+00$ & $8.50 \mathrm{E}-01$ & $6.59 \mathrm{E}+00$ & $4.43 E+00$ & $5.05 \mathrm{E}+00$ \\
\hline AGR-1 633 & $3.84 \mathrm{E}-01$ & 2.99E-01 & 2.01E-02 & $9.23 \mathrm{E}-03$ & $5.23 \mathrm{E}+00$ & 9.47E-01 & $6.97 \mathrm{E}+00$ & $4.73 \mathrm{E}+00$ & $5.42 \mathrm{E}+00$ \\
\hline AGR-1 623 & 4.19E-01 & 3.64E-01 & 2.21E-02 & 1.10E-02 & $5.47 \mathrm{E}+00$ & $1.07 \mathrm{E}+00$ & $6.95 E+00$ & $4.85 \mathrm{E}+00$ & $5.31 E+00$ \\
\hline AGR-1 613 & 4.52E-01 & 4.53E-01 & 2.37E-02 & $1.31 \mathrm{E}-02$ & $5.53 \mathrm{E}+00$ & $1.19 \mathrm{E}+00$ & $6.47 \mathrm{E}+00$ & 4.77E+00 & 4.97E+00 \\
\hline AGR-1 632 & 3.04E-01 & $1.90 \mathrm{E}-01$ & 1.75E-02 & $6.46 \mathrm{E}-03$ & 4.77E+00 & 7.83E-01 & $7.48 \mathrm{E}+00$ & $4.90 \mathrm{E}+00$ & $5.78 \mathrm{E}+00$ \\
\hline AGR-1 622 & 3.36E-01 & $2.36 \mathrm{E}-01$ & $1.96 \mathrm{E}-02$ & 7.81E-03 & $5.14 \mathrm{E}+00$ & 8.85E-01 & $7.63 \mathrm{E}+00$ & $5.05 E+00$ & $5.87 \mathrm{E}+00$ \\
\hline AGR-1 612 & 3.77E-01 & 3.16E-01 & $2.02 \mathrm{E}-02$ & 9.97E-03 & $5.31 \mathrm{E}+00$ & $1.01 \mathrm{E}+00$ & $7.24 \mathrm{E}+00$ & $4.95 \mathrm{E}+00$ & $5.55 \mathrm{E}+00$ \\
\hline AGR-1 641 & 3.63E-01 & 2.60E-01 & $1.92 \mathrm{E}-02$ & 8.43E-03 & $4.94 \mathrm{E}+00$ & 8.29E-01 & $6.57 \mathrm{E}+00$ & 4.29E+00 & $4.88 \mathrm{E}+00$ \\
\hline
\end{tabular}

Table 4. Decay corrected measured Activities for AGR-1 Capsule 5

\begin{tabular}{|c|c|c|c|c|c|c|c|c|c|}
\hline \multirow[b]{2}{*}{ Compact ID } & \multicolumn{9}{|c|}{ Activity (Ci) } \\
\hline & Cs-137 & Cs-134 & Sb-125 & Eu-154 & $\begin{array}{l}\text { Pr-144/ } \\
\text { Ce-144 }\end{array}$ & $\begin{array}{l}\text { Rh-106/ } \\
\text { Ru-106 }\end{array}$ & Zr-95 & Ru-103 & Ce-141 \\
\hline AGR-1543 & 4.76E-01 & $5.32 \mathrm{E}-01$ & $2.39 \mathrm{E}-02$ & $1.51 \mathrm{E}-02$ & $5.84 \mathrm{E}+00$ & $1.34 \mathrm{E}+00$ & $6.74 \mathrm{E}+00$ & $5.09 \mathrm{E}+00$ & $5.25 \mathrm{E}+00$ \\
\hline AGR-1 533 & 4.78E-01 & 5.37E-01 & $2.49 \mathrm{E}-02$ & 1.47E-02 & $5.98 \mathrm{E}+00$ & $1.39 \mathrm{E}+00$ & $6.96 \mathrm{E}+00$ & $5.23 \mathrm{E}+00$ & $5.39 \mathrm{E}+00$ \\
\hline AGR-1 523 & 4.92E-01 & $5.80 \mathrm{E}-01$ & $2.48 \mathrm{E}-02$ & $1.52 \mathrm{E}-02$ & $5.99 \mathrm{E}+00$ & $1.46 E+00$ & $6.75 E+00$ & $5.16 \mathrm{E}+00$ & $5.15 \mathrm{E}+00$ \\
\hline AGR-1513 & $5.15 \mathrm{E}-01$ & 6.69E-01 & $2.59 \mathrm{E}-02$ & $1.76 \mathrm{E}-02$ & $5.84 \mathrm{E}+00$ & $1.57 \mathrm{E}+00$ & $6.17 \mathrm{E}+00$ & $5.06 \mathrm{E}+00$ & $4.72 E+00$ \\
\hline AGR-1 542 & 4.00E-01 & $3.67 E-01$ & $2.10 \mathrm{E}-02$ & $1.14 \mathrm{E}-02$ & $5.75 \mathrm{E}+00$ & $1.14 \mathrm{E}+00$ & $7.99 \mathrm{E}+00$ & $5.42 \mathrm{E}+00$ & $6.10 \mathrm{E}+00$ \\
\hline AGR-1 532 & $3.98 \mathrm{E}-01$ & $3.56 \mathrm{E}-01$ & $2.17 E-02$ & $1.10 \mathrm{E}-02$ & $5.93 \mathrm{E}+00$ & $1.18 \mathrm{E}+00$ & $8.52 E+00$ & $5.74 E+00$ & $6.58 \mathrm{E}+00$ \\
\hline AGR-1 522 & 4.15E-01 & 3.92E-01 & $2.22 \mathrm{E}-02$ & $1.21 \mathrm{E}-02$ & $6.09 \mathrm{E}+00$ & $1.25 \mathrm{E}+00$ & $8.43 \mathrm{E}+00$ & $5.77 \mathrm{E}+00$ & $6.34 \mathrm{E}+00$ \\
\hline AGR-1 512 & 4.44E-01 & 4.81E-01 & $2.37 E-02$ & $1.39 \mathrm{E}-02$ & $6.00 \mathrm{E}+00$ & $1.34 \mathrm{E}+00$ & $7.61 \mathrm{E}+00$ & $5.38 \mathrm{E}+00$ & $5.70 \mathrm{E}+00$ \\
\hline AGR-1 541 & 4.65E-01 & 5.09E-01 & $2.38 \mathrm{E}-02$ & $1.48 \mathrm{E}-02$ & $5.76 \mathrm{E}+00$ & $1.29 \mathrm{E}+00$ & $6.63 E+00$ & $4.82 E+00$ & $4.98 \mathrm{E}+00$ \\
\hline AGR-1 531 & $4.70 \mathrm{E}-01$ & $5.15 \mathrm{E}-01$ & $2.35 \mathrm{E}-02$ & $1.47 \mathrm{E}-02$ & $5.90 \mathrm{E}+00$ & $1.34 \mathrm{E}+00$ & $6.83 \mathrm{E}+00$ & $4.98 \mathrm{E}+00$ & $5.18 \mathrm{E}+00$ \\
\hline AGR-1 521 & 4.83E-01 & $5.58 \mathrm{E}-01$ & 2.49E-02 & $1.53 \mathrm{E}-02$ & $5.94 \mathrm{E}+00$ & $1.41 \mathrm{E}+00$ & $6.70 E+00$ & $5.01 \mathrm{E}+00$ & $5.05 \mathrm{E}+00$ \\
\hline AGR-1 511 & $5.06 \mathrm{E}-01$ & $6.42 \mathrm{E}-01$ & $2.51 \mathrm{E}-02$ & $1.74 \mathrm{E}-02$ & $5.78 E+00$ & $1.51 E+00$ & $6.09 \mathrm{E}+00$ & $4.80 \mathrm{E}+00$ & $4.57 E+00$ \\
\hline
\end{tabular}


Rev. 4

Analysis of Individual Compact Fission Product Inventory and Burnup for the AGR-1 TRISO Experiment using

Title: Gamma Spectrometry

ECAR No.: 1682

ECAR Rev. No.: 3

Project File No.: 23843

Date: $05 / 21 / 2014$

Table 5. Decay corrected measured Activities for AGR-1 Capsule 4

\begin{tabular}{|c|c|c|c|c|c|c|c|c|c|}
\hline \multirow{2}{*}{ Compact ID } & \multicolumn{7}{|c|}{ Activity (Ci) } \\
\cline { 2 - 11 } & Cs-137 & Cs-134 & Sb-125 & Eu-154 & $\begin{array}{c}\text { Pr-144/ } \\
\text { Ce-144 }\end{array}$ & $\begin{array}{c}\text { Rh-106/ } \\
\text { Ru-106 }\end{array}$ & Zr-05 & Ru-103 & Ce-141 \\
\hline AGR-1 443 & $5.45 \mathrm{E}-01$ & $7.50 \mathrm{E}-01$ & $2.74 \mathrm{E}-02$ & $1.93 \mathrm{E}-02$ & $6.08 \mathrm{E}+00$ & $1.72 \mathrm{E}+00$ & $6.06 \mathrm{E}+00$ & $5.31 \mathrm{E}+00$ & $4.71 \mathrm{E}+00$ \\
\hline AGR-1 433 & $5.45 \mathrm{E}-01$ & $7.39 \mathrm{E}-01$ & $2.73 \mathrm{E}-02$ & $1.91 \mathrm{E}-02$ & $6.28 \mathrm{E}+00$ & $1.74 \mathrm{E}+00$ & $6.39 \mathrm{E}+00$ & $5.47 \mathrm{E}+00$ & $4.96 \mathrm{E}+00$ \\
\hline AGR-1 423 & $5.49 \mathrm{E}-01$ & $7.56 \mathrm{E}-01$ & $2.78 \mathrm{E}-02$ & $1.98 \mathrm{E}-02$ & $6.31 \mathrm{E}+00$ & $1.76 \mathrm{E}+00$ & $6.33 \mathrm{E}+00$ & $5.46 \mathrm{E}+00$ & $4.94 \mathrm{E}+00$ \\
\hline AGR-1 413 & $5.45 \mathrm{E}-01$ & $7.69 \mathrm{E}-01$ & $2.66 \mathrm{E}-02$ & $2.03 \mathrm{E}-02$ & $6.00 \mathrm{E}+00$ & $1.71 \mathrm{E}+00$ & $5.82 \mathrm{E}+00$ & $5.07 \mathrm{E}+00$ & $4.52 \mathrm{E}+00$ \\
\hline AGR-1 442 & $4.74 \mathrm{E}-01$ & $5.46 \mathrm{E}-01$ & $2.46 \mathrm{E}-02$ & $1.61 \mathrm{E}-02$ & $6.27 \mathrm{E}+00$ & $1.45 \mathrm{E}+00$ & $7.42 \mathrm{E}+00$ & $5.52 \mathrm{E}+00$ & $5.64 \mathrm{E}+00$ \\
\hline AGR-1 432 & $4.63 \mathrm{E}-01$ & $5.16 \mathrm{E}-01$ & $2.44 \mathrm{E}-02$ & $1.53 \mathrm{E}-02$ & $6.50 \mathrm{E}+00$ & $1.46 \mathrm{E}+00$ & $8.02 \mathrm{E}+00$ & $5.87 \mathrm{E}+00$ & $6.00 \mathrm{E}+00$ \\
\hline AGR-1 422 & $4.54 \mathrm{E}-01$ & $5.16 \mathrm{E}-01$ & $2.47 \mathrm{E}-02$ & $1.46 \mathrm{E}-02$ & $6.33 \mathrm{E}+00$ & $1.46 \mathrm{E}+00$ & $7.74 \mathrm{E}+00$ & $5.69 \mathrm{E}+00$ & $5.72 \mathrm{E}+00$ \\
\hline AGR-1 412 & $4.67 \mathrm{E}-01$ & $5.76 \mathrm{E}-01$ & $2.42 \mathrm{E}-02$ & $1.65 \mathrm{E}-02$ & $5.84 \mathrm{E}+00$ & $1.43 \mathrm{E}+00$ & $6.65 \mathrm{E}+00$ & $5.09 \mathrm{E}+00$ & $5.01 \mathrm{E}+00$ \\
\hline AGR-1 441 & $4.93 \mathrm{E}-01$ & $6.57 \mathrm{E}-01$ & $2.41 \mathrm{E}-02$ & $1.71 \mathrm{E}-02$ & $5.61 \mathrm{E}+00$ & $1.52 \mathrm{E}+00$ & $5.62 \mathrm{E}+00$ & $4.65 \mathrm{E}+00$ & $4.29 \mathrm{E}+00$ \\
\hline AGR-1 431 & $4.77 \mathrm{E}-01$ & $6.20 \mathrm{E}-01$ & $2.45 \mathrm{E}-02$ & $1.65 \mathrm{E}-02$ & $5.61 \mathrm{E}+00$ & $1.46 \mathrm{E}+00$ & $5.68 \mathrm{E}+00$ & $4.63 \mathrm{E}+00$ & $4.22 \mathrm{E}+00$ \\
\hline AGR-1 421 & $4.83 \mathrm{E}-01$ & $6.40 \mathrm{E}-01$ & $2.40 \mathrm{E}-02$ & $1.71 \mathrm{E}-02$ & $5.64 \mathrm{E}+00$ & $1.51 \mathrm{E}+00$ & $5.64 \mathrm{E}+00$ & $4.65 \mathrm{E}+00$ & $4.20 \mathrm{E}+00$ \\
\hline AGR-1 411 & $5.10 \mathrm{E}-01$ & $7.48 \mathrm{E}-01$ & $2.47 \mathrm{E}-02$ & $1.91 \mathrm{E}-02$ & $5.46 \mathrm{E}+00$ & $1.64 \mathrm{E}+00$ & $5.10 \mathrm{E}+00$ & $4.61 \mathrm{E}+00$ & $3.89 \mathrm{E}+00$ \\
\hline
\end{tabular}

Table 6. Decay corrected measured Activities for AGR-1 Capsule 3

\begin{tabular}{|c|c|c|c|c|c|c|c|c|c|}
\hline \multirow{2}{*}{ Compact ID } & \multicolumn{70}{|c|}{ Activity (Ci) } \\
\cline { 2 - 11 } & Cs-137 & Cs-134 & Sb-125 & Eu-154 & $\begin{array}{c}\text { Pr-144/ } \\
\text { Ce-144 }\end{array}$ & $\begin{array}{c}\text { Rh-106/ } \\
\text { Ru-106 }\end{array}$ & Zr-95 & Ru-103 & Ce-141 \\
\hline AGR-1 343 & $5.45 \mathrm{E}-01$ & $8.06 \mathrm{E}-01$ & $2.73 \mathrm{E}-02$ & $2.05 \mathrm{E}-02$ & $5.84 \mathrm{E}+00$ & $1.78 \mathrm{E}+00$ & $5.62 \mathrm{E}+00$ & $5.03 \mathrm{E}+00$ & $4.29 \mathrm{E}+00$ \\
\hline AGR-1 333 & $5.30 \mathrm{E}-01$ & $7.58 \mathrm{E}-01$ & $2.70 \mathrm{E}-02$ & $1.93 \mathrm{E}-02$ & $5.90 \mathrm{E}+00$ & $1.72 \mathrm{E}+00$ & $5.84 \mathrm{E}+00$ & $5.06 \mathrm{E}+00$ & $4.49 \mathrm{E}+00$ \\
\hline AGR-1 323 & $5.59 \mathrm{E}-01$ & $7.97 \mathrm{E}-01$ & $2.86 \mathrm{E}-02$ & $2.03 \mathrm{E}-02$ & $6.24 \mathrm{E}+00$ & $1.80 \mathrm{E}+00$ & $6.19 \mathrm{E}+00$ & $5.32 \mathrm{E}+00$ & $4.76 \mathrm{E}+00$ \\
\hline AGR-1 313 & $5.45 \mathrm{E}-01$ & $8.09 \mathrm{E}-01$ & $2.68 \mathrm{E}-02$ & $2.00 \mathrm{E}-02$ & $5.83 \mathrm{E}+00$ & $1.80 \mathrm{E}+00$ & $5.62 \mathrm{E}+00$ & $5.09 \mathrm{E}+00$ & $4.39 \mathrm{E}+00$ \\
\hline AGR-1 342 & $4.75 \mathrm{E}-01$ & $5.97 \mathrm{E}-01$ & $2.44 \mathrm{E}-02$ & $1.64 \mathrm{E}-02$ & $6.07 \mathrm{E}+00$ & $1.52 \mathrm{E}+00$ & $6.98 \mathrm{E}+00$ & $5.37 \mathrm{E}+00$ & $5.16 \mathrm{E}+00$ \\
\hline AGR-1 332 & $4.55 \mathrm{E}-01$ & $5.39 \mathrm{E}-01$ & $2.46 \mathrm{E}-02$ & $1.50 \mathrm{E}-02$ & $6.15 \mathrm{E}+00$ & $1.45 \mathrm{E}+00$ & $7.42 \mathrm{E}+00$ & $5.38 \mathrm{E}+00$ & $5.53 \mathrm{E}+00$ \\
\hline AGR-1 322 & $4.40 \mathrm{E}-01$ & $5.21 \mathrm{E}-01$ & $2.37 \mathrm{E}-02$ & $1.46 \mathrm{E}-02$ & $5.95 \mathrm{E}+00$ & $1.40 \mathrm{E}+00$ & $7.22 \mathrm{E}+00$ & $5.27 \mathrm{E}+00$ & $5.31 \mathrm{E}+00$ \\
\hline AGR-1 312 & $4.48 \mathrm{E}-01$ & $5.69 \mathrm{E}-01$ & $2.40 \mathrm{E}-02$ & $1.57 \mathrm{E}-02$ & $5.66 \mathrm{E}+00$ & $1.42 \mathrm{E}+00$ & $6.48 \mathrm{E}+00$ & $4.93 \mathrm{E}+00$ & $4.80 \mathrm{E}+00$ \\
\hline AGR-1 341 & $4.99 \mathrm{E}-01$ & $7.10 \mathrm{E}-01$ & $2.51 \mathrm{E}-02$ & $1.85 \mathrm{E}-02$ & $5.40 \mathrm{E}+00$ & $1.59 \mathrm{E}+00$ & $5.27 \mathrm{E}+00$ & $4.55 \mathrm{E}+00$ & $3.81 \mathrm{E}+00$ \\
\hline AGR-1 331 & $5.16 \mathrm{E}-01$ & $7.05 \mathrm{E}-01$ & $2.64 \mathrm{E}-02$ & $1.82 \mathrm{E}-02$ & $5.90 \mathrm{E}+00$ & $1.63 \mathrm{E}+00$ & $5.93 \mathrm{E}+00$ & $4.86 \mathrm{E}+00$ & $4.47 \mathrm{E}+00$ \\
\hline AGR-1 321 & $5.08 \mathrm{E}-01$ & $6.89 \mathrm{E}-01$ & $2.47 \mathrm{E}-02$ & $1.80 \mathrm{E}-02$ & $5.84 \mathrm{E}+00$ & $1.60 \mathrm{E}+00$ & $5.90 \mathrm{E}+00$ & $4.81 \mathrm{E}+00$ & $4.33 \mathrm{E}+00$ \\
\hline AGR-1 311 & $5.06 \mathrm{E}-01$ & $7.24 \mathrm{E}-01$ & $2.51 \mathrm{E}-02$ & $1.84 \mathrm{E}-02$ & $5.50 \mathrm{E}+00$ & $1.62 \mathrm{E}+00$ & $5.33 \mathrm{E}+00$ & $4.59 \mathrm{E}+00$ & $3.96 \mathrm{E}+00$ \\
\hline
\end{tabular}


Rev. 4

Analysis of Individual Compact Fission Product Inventory and Burnup for the AGR-1 TRISO Experiment using

Title: Gamma Spectrometry

ECAR No.: 1682

ECAR Rev. No.: 3

Project File No.: 23843

Date: $05 / 21 / 2014$

Table 7. Decay corrected measured Activities for AGR-1 Capsule 2

\begin{tabular}{|c|c|c|c|c|c|c|c|c|c|}
\hline \multirow{2}{*}{ Compact ID } & \multicolumn{9}{|c|}{ Activity (Ci) } \\
\cline { 2 - 11 } & Cs-137 & Cs-134 & Sb-125 & Eu-154 & $\begin{array}{c}\text { Pr-144/ } \\
\text { Ce-144 }\end{array}$ & $\begin{array}{c}\text { Rh-106/ } \\
\text { Ru-106 }\end{array}$ & Zr-95 & Ru-103 & Ce-141* \\
\hline AGR-1 243 & $5.31 \mathrm{E}-01$ & $7.55 \mathrm{E}-01$ & $2.65 \mathrm{E}-02$ & $1.84 \mathrm{E}-02$ & $5.85 \mathrm{E}+00$ & $1.75 \mathrm{E}+00$ & $5.98 \mathrm{E}+00$ & $5.29 \mathrm{E}+00$ & -- \\
\hline AGR-1 233 & $5.17 \mathrm{E}-01$ & $6.96 \mathrm{E}-01$ & $2.58 \mathrm{E}-02$ & $1.74 \mathrm{E}-02$ & $6.02 \mathrm{E}+00$ & $1.68 \mathrm{E}+00$ & $6.35 \mathrm{E}+00$ & $5.23 \mathrm{E}+00$ & -- \\
\hline AGR-1 223 & $5.04 \mathrm{E}-01$ & $6.60 \mathrm{E}-01$ & $2.54 \mathrm{E}-02$ & $1.67 \mathrm{E}-02$ & $5.92 \mathrm{E}+00$ & $1.62 \mathrm{E}+00$ & $6.37 \mathrm{E}+00$ & $5.17 \mathrm{E}+00$ & -- \\
\hline AGR-1 213 & $5.14 \mathrm{E}-01$ & $6.86 \mathrm{E}-01$ & $2.51 \mathrm{E}-02$ & $1.77 \mathrm{E}-02$ & $5.64 \mathrm{E}+00$ & $1.60 \mathrm{E}+00$ & $5.34 \mathrm{E}+00$ & $3.87 \mathrm{E}+00$ & -- \\
\hline AGR-1 242 & $4.50 \mathrm{E}-01$ & $5.34 \mathrm{E}-01$ & $2.31 \mathrm{E}-02$ & $1.47 \mathrm{E}-02$ & $5.71 \mathrm{E}+00$ & $1.41 \mathrm{E}+00$ & $6.22 \mathrm{E}+00$ & $4.35 \mathrm{E}+00$ & -- \\
\hline AGR-1 232 & $4.75 \mathrm{E}-01$ & $5.19 \mathrm{E}-01$ & $2.62 \mathrm{E}-02$ & $1.45 \mathrm{E}-02$ & $6.46 \mathrm{E}+00$ & $1.51 \mathrm{E}+00$ & $7.49 \mathrm{E}+00$ & $5.30 \mathrm{E}+00$ & -- \\
\hline AGR-1 222 & $4.35 \mathrm{E}-01$ & $4.58 \mathrm{E}-01$ & $2.35 \mathrm{E}-02$ & $1.30 \mathrm{E}-02$ & $5.98 \mathrm{E}+00$ & $1.37 \mathrm{E}+00$ & $7.04 \mathrm{E}+00$ & $4.69 \mathrm{E}+00$ & -- \\
\hline AGR-1 212 & $4.48 \mathrm{E}-01$ & $5.02 \mathrm{E}-01$ & $2.36 \mathrm{E}-02$ & $1.43 \mathrm{E}-02$ & $5.82 \mathrm{E}+00$ & $1.38 \mathrm{E}+00$ & $6.59 \mathrm{E}+00$ & $4.54 \mathrm{E}+00$ & -- \\
\hline AGR-1 241 & $5.14 \mathrm{E}-01$ & $6.94 \mathrm{E}-01$ & $2.53 \mathrm{E}-02$ & $1.79 \mathrm{E}-02$ & $5.58 \mathrm{E}+00$ & $1.60 \mathrm{E}+00$ & $5.18 \mathrm{E}+00$ & $3.91 \mathrm{E}+00$ & -- \\
\hline AGR-1 231 & $4.98 \mathrm{E}-01$ & $6.29 \mathrm{E}-01$ & $2.48 \mathrm{E}-02$ & $1.64 \mathrm{E}-02$ & $5.77 \mathrm{E}+00$ & $1.54 \mathrm{E}+00$ & $5.61 \mathrm{E}+00$ & $4.10 \mathrm{E}+00$ & -- \\
\hline AGR-1 221 & $4.94 \mathrm{E}-01$ & $6.11 \mathrm{E}-01$ & $2.51 \mathrm{E}-02$ & $1.61 \mathrm{E}-02$ & $5.77 \mathrm{E}+00$ & $1.52 \mathrm{E}+00$ & $5.70 \mathrm{E}+00$ & $3.95 \mathrm{E}+00$ & -- \\
\hline AGR-1 211 & $4.99 \mathrm{E}-01$ & $6.34 \mathrm{E}-01$ & $2.46 \mathrm{E}-02$ & $1.65 \mathrm{E}-02$ & $5.64 \mathrm{E}+00$ & $1.51 \mathrm{E}+00$ & $5.45 \mathrm{E}+00$ & $4.04 \mathrm{E}+00$ & -- \\
\hline
\end{tabular}

* Capsule 2 was the last capsule scanned; by the time it was scanned all the Ce-141 had decayed away ( $>13$ half lives had passed)

Table 8. Decay corrected measured Activities for AGR-1 Capsule 1

\begin{tabular}{|c|c|c|c|c|c|c|c|c|c|}
\hline \multirow[b]{2}{*}{ Compact ID } & \multicolumn{9}{|c|}{ Activity (Ci) } \\
\hline & Cs-137 & Cs-134 & Sb-125 & Eu-154 & $\begin{array}{l}\text { Pr-144/ } \\
\text { Ce-144 }\end{array}$ & $\begin{array}{l}\text { Rh-106/ } \\
\text { Ru-106 }\end{array}$ & Zr-95 & Ru-103 & Ce-141 \\
\hline AGR-1 143 & 4.62E-01 & $5.76 \mathrm{E}-01$ & 2.36E-02 & $1.58 \mathrm{E}-02$ & $5.29 \mathrm{E}+00$ & $1.36 \mathrm{E}+00$ & $5.63 \mathrm{E}+00$ & $4.59 \mathrm{E}+00$ & $4.30 \mathrm{E}+00$ \\
\hline AGR-1 133 & 4.55E-01 & $5.13 \mathrm{E}-01$ & 2.30E-02 & $1.45 \mathrm{E}-02$ & $5.47 E+00$ & $1.27 E+00$ & $6.20 \mathrm{E}+00$ & $4.76 \mathrm{E}+00$ & $4.77 \mathrm{E}+00$ \\
\hline AGR-1 123 & $4.42 \mathrm{E}-01$ & 4.70E-01 & $2.32 \mathrm{E}-02$ & $1.35 \mathrm{E}-02$ & $5.53 \mathrm{E}+00$ & $1.23 \mathrm{E}+00$ & $6.45 \mathrm{E}+00$ & $4.81 \mathrm{E}+00$ & $4.98 \mathrm{E}+00$ \\
\hline AGR-1 113 & 4.42E-01 & 4.71E-01 & 2.19E-02 & $1.36 \mathrm{E}-02$ & $5.32 \mathrm{E}+00$ & $1.19 \mathrm{E}+00$ & $6.12 \mathrm{E}+00$ & $4.54 \mathrm{E}+00$ & $4.69 \mathrm{E}+00$ \\
\hline AGR-1 142 & $4.21 \mathrm{E}-01$ & 4.45E-01 & $2.29 \mathrm{E}-02$ & $1.33 \mathrm{E}-02$ & $5.59 \mathrm{E}+00$ & $1.22 \mathrm{E}+00$ & $6.99 \mathrm{E}+00$ & $5.08 \mathrm{E}+00$ & $5.39 \mathrm{E}+00$ \\
\hline AGR-1 132 & $3.92 \mathrm{E}-01$ & 3.66E-01 & 2.20E-02 & 1.07E-02 & $5.63 \mathrm{E}+00$ & $1.13 \mathrm{E}+00$ & $7.53 E+00$ & $5.24 \mathrm{E}+00$ & $5.68 \mathrm{E}+00$ \\
\hline AGR-1 122 & $3.73 \mathrm{E}-01$ & 3.27E-01 & 2.15E-02 & 9.96E-03 & $5.49 \mathrm{E}+00$ & $1.06 \mathrm{E}+00$ & $7.58 \mathrm{E}+00$ & $5.14 E+00$ & $5.68 \mathrm{E}+00$ \\
\hline AGR-1 112 & $3.76 \mathrm{E}-01$ & 3.32E-01 & 2.00E-02 & $1.04 \mathrm{E}-02$ & $5.24 E+00$ & 9.97E-01 & $6.97 E+00$ & $4.74 \mathrm{E}+00$ & $5.27 \mathrm{E}+00$ \\
\hline AGR-1 141 & 4.67E-01 & $5.47 \mathrm{E}-01$ & $2.36 \mathrm{E}-02$ & $1.52 \mathrm{E}-02$ & $5.52 \mathrm{E}+00$ & $1.33 \mathrm{E}+00$ & $5.95 \mathrm{E}+00$ & $4.58 \mathrm{E}+00$ & $4.58 \mathrm{E}+00$ \\
\hline AGR-1 131 & 4.46E-01 & 4.74E-01 & $2.26 \mathrm{E}-02$ & $1.35 \mathrm{E}-02$ & $5.58 \mathrm{E}+00$ & $1.24 \mathrm{E}+00$ & $6.37 \mathrm{E}+00$ & $4.69 \mathrm{E}+00$ & $4.88 \mathrm{E}+00$ \\
\hline AGR-1 121 & 4.27E-01 & 4.30E-01 & 2.19E-02 & $1.22 \mathrm{E}-02$ & $5.49 \mathrm{E}+00$ & $1.16 \mathrm{E}+00$ & $6.53 \mathrm{E}+00$ & $4.68 \mathrm{E}+00$ & $4.87 \mathrm{E}+00$ \\
\hline AGR-1 111 & $4.26 \mathrm{E}-01$ & 4.30E-01 & $2.22 \mathrm{E}-02$ & $1.28 \mathrm{E}-02$ & $5.30 \mathrm{E}+00$ & $1.11 \mathrm{E}+00$ & $6.15 \mathrm{E}+00$ & $4.41 E+00$ & $4.59 \mathrm{E}+00$ \\
\hline
\end{tabular}

Several of the measured activities from the AGR-1 compacts are compared with the calculated activities from Reference 4 in Fig. 4. It should be noted that in Fig. 4 the C/E value for Cs-137 stays very close to 1 (test average C/E 1.0013 $( \pm 0.0319)$ ), which indicates its behavior was captured very well by simulation and should act as a reliable link between measured activity and burnup. The simulated activity of Cs-134 closely matches the measured activities with a test average $\mathrm{C} / \mathrm{E}$ of $1.0190( \pm 0.0506)$ for all the compacts. The test average C/E for Eu-154 is 1.1835( \pm 0.0525$)$, indicating a bias between the simulation and the measurement. The activity of Sb-125 also shows a bias between simulation and measurement with a compact average $\mathrm{C} / \mathrm{E}$ of $1.3955( \pm 0.0860)$. The bias in these values may possibly be due to a variety of sources including nuclear data or changing irradiation conditions. The simulated activities of Ce-144 and Ru-106 agree well with PGS measurements. The average C/E for Ce-144 as measured by Pr-144 activity is 0.9939 ( \pm 0.0367$)$, and the average C/E for Ru106 as measured by $\mathrm{Rh}-106$ is $1.0404( \pm 0.0432)$. 
Analysis of Individual Compact Fission Product Inventory and Burnup for the AGR-1 TRISO Experiment using Title: Gamma Spectrometry

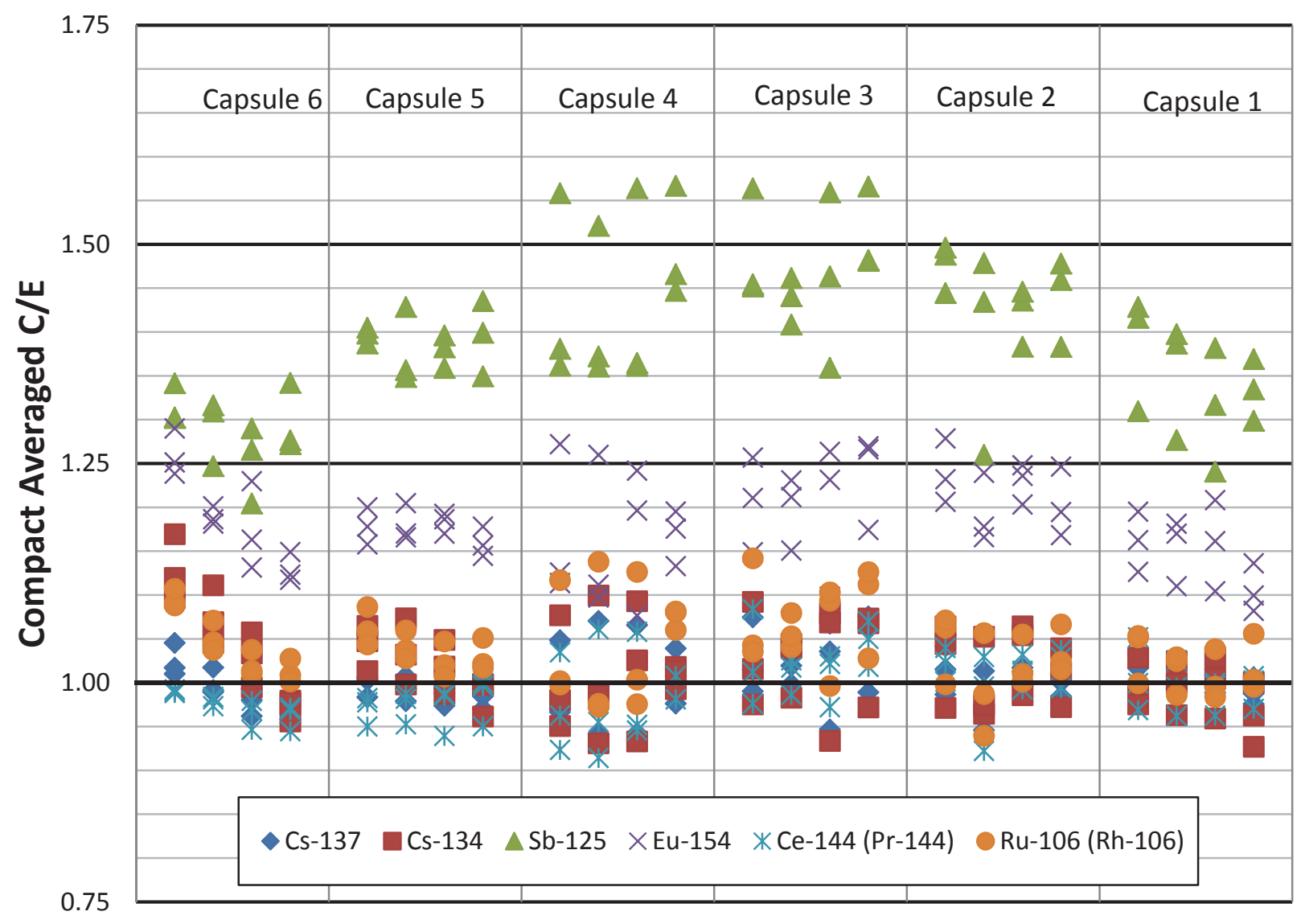

\section{Experiment Axial Position}

Fig. 4. Simulated to measured activity ratio for several different fission products for each fuel compact in the AGR-1 test train. Fuel compacts are plotted by vertical position in the AGR-1 test train, with the top of the test train at the left and the bottom at the right. There are three compacts at each axial location.

\subsection{Burnup Evaluation from PGS Scans}

In order to convert a measured fission product activity into a burnup value, some relationship between the activity of an isotope or the ratio of activities must be established through calculation or simulation. Burnup assessment by both the direct activity measurement method and the activity ratio method were explored in this work. The absolute activity needed in the direct method requires careful evaluation of the efficiency of the detection system. The activity ratio method requires only a relative efficiency detector system calibration which is much easier to perform. However, for high burnup experiments, such as AGR-1, the inventory of a fission product is complicated by the depletion of U-235, the breeding of Pu-239, and shifts in the neutron energy spectrum as burnup increases. These same complicating factors also make using shielded fission productto-direct fission product activity ratios difficult in high burnup situations.2 The production of shielded fission products relies on a neutron capture reaction taking place in a previously produced fission product. This complex process can place too much uncertainty on the creation of a function that could relate burnup to a ratio of a shielded fission product (e.g. Cs-134 or Eu-154) and a direct fission product (e.g. Cs-137, Sb-125) in high burnup systems (i.e. greater than 5\% FIMA). However, the simulations performed in Reference 4 appear to have faithfully reproduced the end of irradiation activities for several isotopes including Cs-134, so it may still be possible to find a relationship between the Cs-134 to Cs-137 ratio and burnup. 
Analysis of Individual Compact Fission Product Inventory and Burnup for the AGR-1 TRISO Experiment using Title: Gamma Spectrometry

A usable fission product also needs to increase monotonically in activity during the entire course of an irradiation. There were 11 fission products stated in Section 4.4 that were detectable in the PGS scans. During the course of the AGR-1 irradiation of 620 effective full power days that spanned nearly 3 calendar years, several of these isotopes achieved an equilibrium activity (e.g. Y-91, Zr-95) during the irradiation. This equilibrium behavior makes them unsuitable for burnup measurements. Of all the fission products detected in the PGS scans, Cs-137 is the optimum burnup indicator isotope for several reasons. It is a direct fission product meaning its production in the fuel is roughly proportional neutron fluence which is proportional to burnup. It is long lived $\left(\mathrm{T}_{1 / 2}=30.1\right.$ years) enough to keep increasing in activity linearly with time and burnup throughout the course of the long AGR-1 irradiation. It does not have a significant neutron absorption cross section that would complicate the simulation of its inventory. It also has a similar fission yield between U-235 and Pu- 239 which also reduces the complexity of simulation for this isotope.

Detailed production/depletion calculations of the AGR-1 experiment were performed in which all of the as-run reactor power conditions were considered. 4 The results of this simulation can be used to link the measured compact activities to burnup. The simulation coupled Monte Carlo neutron transport in MCNP5 with depletion calculations in ORIGEN2.2 using the INL developed code JMOCUP (Jim's MCNP-ORIGEN2 Coupled Utility Program). JMOCUP uses data from MCNP to calculate one-group cross sections for use in ORIGEN depletion calculations. In this simulation each fuel compact was split into two calculation nodes. The results of this simulation included both actinide inventories and fission product inventories. The actinide inventories were used to calculate burnup in FIMA using Equation 1.

$$
B U=\frac{N_{U-235, i}+N_{U-238, i}-N_{U-235, f}-N_{U-238, f}-N_{A c t, f}}{N_{U-235, i}+N_{U-238, i}}
$$

Where $N_{U-235, i}$ is the initial number of U-235 atoms, $N_{U-238, i}$ is the initial number of U-238 atoms, $N_{U-235, f}$ is the final number of U-235 atoms, $N_{U-238, f}$ is the final number of U-238 atoms, and $N_{A c t, f}$ is the final number of actinide atoms created by neutron capture of U-235 and U-238 atoms. The calculation node burnup values from Equation 1 were averaged for each fuel compact to get compact average burnups that are used for comparison to burnup measured from the detected compact fission product inventory.

Using the fission product inventory results of the simulation in Reference 4, a relationship was developed between the final predicted Cs-137 activities of the different calculation nodes and the burnup of each node. A linear regression was fit to the data to create a function relating fuel compact averaged Cs-137 inventory to fuel burnup in \% FIMA. This relationship is given in Equation 2.

$$
B U=37.75\left(A_{C s-137}\right)-0.8356
$$

Where $B U$ is the burnup of the fuel in \% FIMA, and $A_{C s-137}$ is the total compact activity of Cs- 137 in Curies. This relationship uses a part of the results from simulation to relate fission product activity to burnup, and a different part of the same simulations are used to predict the burnup of each fuel compact. This may seem like an over reliance on the complex simulation; however, to measure burnup from a fission product inventory requires some sort of correlation between an amount of a fission product and burnup. Fortunately the activity to burnup relationship for Cs-137 can also be expressed through a series of analytical expressions set out in Reference 14 and repeated here in Equations (3) to (6). Equation (3) is the Bateman equation for Cs- 137 where $\mathrm{N}_{\mathrm{Cs}-137}$ is the number of Cs-137 atoms, $\lambda_{\mathrm{Cs}-137}$ is the decay constant of Cs-137, $\gamma_{\mathrm{Cs}-137}$ is the fission yield of Cs-137, $\bar{\sigma}_{f}$ is the spectrum averaged fission cross section for the system under consideration, $\bar{\phi}_{n}$ is the neutron flux, and $\mathrm{N}_{\mathrm{HM}}$ is the number of heavy metal atoms in the system.

$$
\frac{d N_{C s-137}}{d t}=-\lambda_{C s-137} N_{C s-137}+\gamma_{C s-137} \cdot \bar{\sigma}_{f} \bar{\phi}_{n} N_{H M}
$$

Assuming no initial Cs-137 activity in the system Equation (3) can be solved to find Equation (4) assuming the number of fissile atoms remains roughly constant in the system. Using the same fissile atom assumption from Equation (4), an equation for burnup can be derived in the form of Equation (5). Equations (4) and (5) can be combined to get Equation (6), which directly relates burnup to Cs-137 activity.

$$
N_{C s-137}\left(t_{i r r}\right)=\frac{\gamma_{C s-137} \bar{\sigma}_{f} \bar{\phi}_{n} N_{H M}}{\lambda_{C S-137}}\left(1-e^{\left(-\lambda_{C s-137} t_{i r r}\right)}\right)
$$




$$
\begin{gathered}
B U=\frac{\left(\bar{\sigma}_{f} \bar{\phi}_{n} N_{H M}\right) t_{i r r}}{N_{H M}} \\
B U=A_{C s-137}\left(t_{i r r}\right) \frac{t_{i r r}}{\gamma_{C S-137} N_{H M}}\left(1-e^{\left(-\lambda_{C s-13 t_{i r r}}\right)}\right)^{-1}
\end{gathered}
$$

The irradiation times in the AGR-1 experiment are short enough compared to the half-life of Cs-137 that a linear relationship between burnup derived from Equation (5) and Cs-137 Activity derived from Equation (4) can be found using the average irradiation conditions of the AGR-1 experiment $\left(\bar{\sigma}_{f}=152 \mathrm{~b}, \bar{\phi}_{n}=3.2 \times 10^{14} \mathrm{n} / \mathrm{cm}^{2}, \mathrm{~N}_{\mathrm{HM}}=2.28 \times 10^{21}\right.$ atoms Heavy Metal). Using these rather simple equations, the burnup in \%FIMA as a function of Cs-137 activity is shown in Equation (7) where the activity of Cs-137 is in Curies.

$$
B U=35.46\left(A_{C s-137}\right)-0.0063
$$

This burnup relationship compares favorably with the relationship derived from the coupled Monte Carlo / ORIGEN calculations shown in Equation (2). This example shows Cs-137 to be a robust burnup indicator. The simple analytical solutions to the relationship between Cs-137 and burnup were also shown to match other sophisticated simulations for different fuel systems. ${ }^{14}$

A relationship between burnup and the ratio of the activities of Cs-134 and Cs-137 can also be derived from the results of the coupled MCNP / ORIGEN calculations. Only certain fission product inventories change as a function of burnup. For example, a ratio of Cs-137 and Ce-144 would not change with burnup since the inventory of both fission products increases linearly with neutron fluence for irradiation times that are small compared to their half life. Shielded fission products like Cs-134 increase quadratically with neutron fluence for irradiation times that are small compared to their half life. Therefore, the ratio of a shielded to direct fission product will still increase linearly with neutron fluence and burnup under the proper conditions. A linear regression was fit to the compact averaged activity ratio of Cs-134 to Cs-137 values taken from Reference 4. This relationship between activity ratio and burnup for the AGR-1 experiment is shown in the equation below, where $\mathrm{BU}$ is burnup in \% FIMA, $A_{C s-134}$ is the activity of Cs-134 and $A_{C s-137}$ is the activity of Cs-137.

$$
B U=10.4239\left(\frac{A_{C s-134}}{A_{C s-137}}\right)+4.5053
$$

The compact averaged burnup for each AGR-1 compact can now be estimated from the measured Cs-137 activities and the measured Cs-134/Cs-137 activity ratio. The results of the PGS scans were converted to a measured burnup value using Equations (2) and (8). The measured burnups using the activity of Cs-137 were then compared to the burnup calculated by simulation in Reference 4 in Fig. 5. Fig. 5 shows the calculated and measured burnup values for each compact in the AGR-1 test train from top (left) to bottom (right). As the irradiation capsules consisted of a trigonal array of fuel compacts, ${ }^{1}$ there are three compacts at each of the 24 axial levels presented in Fig. 5. Each axial array of fuel in the experiment was labeled Stack 1 to 3. The burnup is higher in Stacks 1 and 3 since they were facing the core as is seen in Fig. 2. The simulated burnup in Stacks 1 and 3 is nearly identical and is represented in Fig. 5 by the filled diamonds. The simulated values between Stacks 1 and 3 are so close together that the diamonds tend to overlap in Fig. 5. However it is possible to see slight differences in the simulated burnup values in the data for Capsule 1. There were some measured burnup differences between Stack 1 and Stack 3 whose burnup is represented as filled squares in Fig. 5. Stack 2 was shielded from the core by the other fuel compacts resulting in a lower burnup in both measurement and predicted by simulation. The measured and simulated burnup of Stack 2 can be seen by the open squares and diamonds in Fig. 5. The majority of uncertainty on the measured burnup is propagated from uncertainty on the efficiency calibration and uncertainty on the Eu-152 calibration sources. The relative uncertainty on the measured burnup values is approximately $5.3 \%$. The peak measured burnup is $20.1 \%$ FIMA for compact $3-2-3$, and the minimum measured burnup is $10.1 \%$ FIMA for compact 6-4-2. A plot similar to Fig. 5 but using the Cs-134 to Cs-137 activity ratio is shown in Fig. 6. The relative uncertainty on the burnup values in Fig. 6 is approximately 3.1\%. The lower relative uncertainty on these values is driven by the simpler efficiency calibration needed by the ratio technique. In the Fig. 6 data, the minimum measured burnup is $10.7 \%$ FIMA for compact $6-4-2$, and the maximum burnup is $20.0 \%$ FIMA for compact 3-1-3. The numeric value of the PGS burnup measurement for each compact and each technique is shown in Table 9 through Table 14 along with the burnup values from simulation. 
Analysis of Individual Compact Fission Product Inventory and Burnup for the AGR-1 TRISO Experiment using Title: Gamma Spectrometry

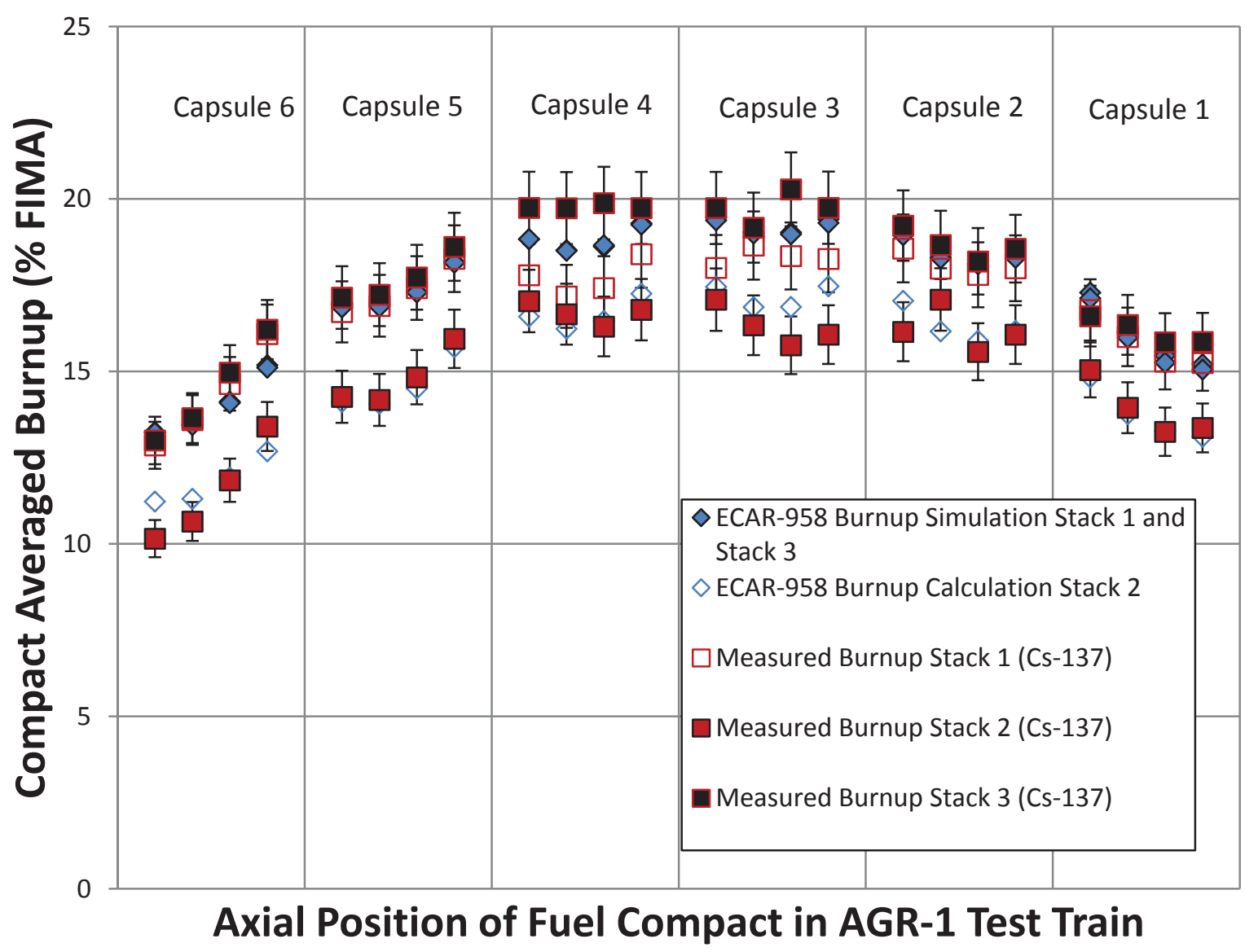

Fig. 5. Comparison of burnup derived from Cs-137 measurements to burnup derived directly from simulations4 
Analysis of Individual Compact Fission Product Inventory and Burnup for the AGR-1 TRISO Experiment using Title: Gamma Spectrometry

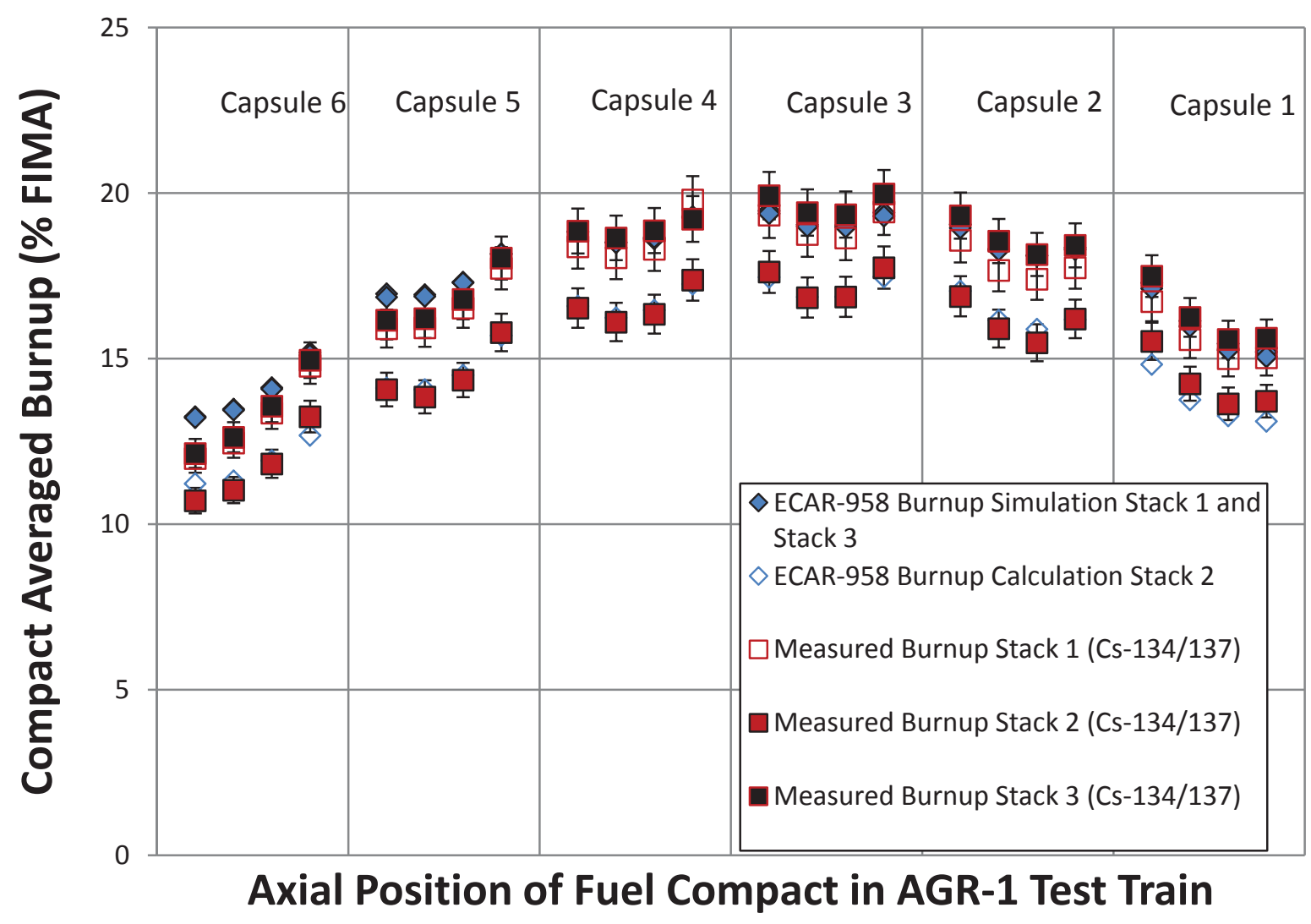

Fig. 6. Comparison of burnup derived from Cs-134/Cs-137 measured activity ratio to burnup derived directly from simulations4 
Rev. 4

Analysis of Individual Compact Fission Product Inventory and Burnup for the AGR-1 TRISO Experiment using Title: Gamma Spectrometry

Table 9. Burnup values for AGR-1 compacts from Capsule 6

\begin{tabular}{|c|c|c|c|}
\hline Compact ID & $\begin{array}{c}\text { Burnup from } \\
\text { Measured Cs-137 } \\
\text { Activity (\% FIMA) }\end{array}$ & $\begin{array}{c}\text { Burnup from } \\
\text { Measured Cs-134/Cs- } \\
\text { 137 Ratio (\% FIMA) }\end{array}$ & $\begin{array}{c}\text { Burnup from Ref. 4 } \\
\text { (ECAR-958) (\% FIMA) }\end{array}$ \\
\hline AGR-1643 & $13.0 \%$ & $12.1 \%$ & $13.25 \%$ \\
\hline AGR-1633 & $13.6 \%$ & $12.6 \%$ & $13.47 \%$ \\
\hline AGR-1623 & $14.9 \%$ & $13.6 \%$ & $14.13 \%$ \\
\hline AGR-1613 & $16.1 \%$ & $15.0 \%$ & $15.18 \%$ \\
\hline AGR-1642 & $10.2 \%$ & $10.7 \%$ & $11.23 \%$ \\
\hline AGR-1632 & $10.7 \%$ & $11.0 \%$ & $11.31 \%$ \\
\hline AGR-1622 & $11.8 \%$ & $11.8 \%$ & $11.93 \%$ \\
\hline AGR-1612 & $13.4 \%$ & $13.3 \%$ & $12.68 \%$ \\
\hline AGR-1641 & $12.8 \%$ & $12.0 \%$ & $13.22 \%$ \\
\hline AGR-1631 & $13.5 \%$ & $12.5 \%$ & $13.45 \%$ \\
\hline AGR-1621 & $14.6 \%$ & $13.4 \%$ & $14.09 \%$ \\
\hline AGR-1611 & $16.0 \%$ & $14.8 \%$ & $15.11 \%$ \\
\hline
\end{tabular}

Table 10. Burnup values for AGR-1 compacts from Capsule 5

\begin{tabular}{|c|c|c|c|}
\hline Compact ID & $\begin{array}{c}\text { Burnup from } \\
\text { Measured Cs-137 } \\
\text { Activity (\% FIMA) }\end{array}$ & $\begin{array}{c}\text { Burnup from } \\
\text { Measured Cs-134/Cs- } \\
\text { 137 Ratio (\% FIMA) }\end{array}$ & $\begin{array}{c}\text { Burnup from Ref. 4 } \\
\text { (ECAR-958) (\% FIMA) }\end{array}$ \\
\hline AGR-1543 & $17.0 \%$ & $16.2 \%$ & $16.96 \%$ \\
\hline AGR-1533 & $17.1 \%$ & $16.2 \%$ & $16.93 \%$ \\
\hline AGR-1523 & $17.6 \%$ & $16.8 \%$ & $17.31 \%$ \\
\hline AGR-1513 & $18.5 \%$ & $18.0 \%$ & $18.17 \%$ \\
\hline AGR-1542 & $14.2 \%$ & $14.1 \%$ & $14.11 \%$ \\
\hline AGR-1532 & $14.1 \%$ & $13.8 \%$ & $14.07 \%$ \\
\hline AGR-1522 & $14.8 \%$ & $14.4 \%$ & $14.50 \%$ \\
\hline AGR-1512 & $15.9 \%$ & $15.8 \%$ & $15.70 \%$ \\
\hline AGR-1541 & $16.6 \%$ & $15.9 \%$ & $16.86 \%$ \\
\hline AGR-1531 & $16.8 \%$ & $15.9 \%$ & $16.88 \%$ \\
\hline AGR-1521 & $17.3 \%$ & $16.5 \%$ & $17.29 \%$ \\
\hline AGR-1511 & $18.1 \%$ & $17.7 \%$ & $18.15 \%$ \\
\hline
\end{tabular}


Rev. 4

Analysis of Individual Compact Fission Product Inventory and Burnup for the AGR-1 TRISO Experiment using

Title: Gamma Spectrometry

Table 11. Burnup values for AGR-1 compacts from Capsule 4

\begin{tabular}{|c|c|c|c|}
\hline Compact ID & $\begin{array}{c}\text { Burnup from } \\
\text { Measured Cs-137 } \\
\text { Activity (\% FIMA) }\end{array}$ & $\begin{array}{c}\text { Burnup from } \\
\text { Measured Cs-134/Cs- } \\
\text { 137 Ratio (\% FIMA) }\end{array}$ & $\begin{array}{c}\text { Burnup from Ref. 4 } \\
\text { (ECAR-958) (\% FIMA) }\end{array}$ \\
\hline AGR-1443 & $19.6 \%$ & $18.9 \%$ & $18.83 \%$ \\
\hline AGR-1433 & $19.6 \%$ & $18.6 \%$ & $18.52 \%$ \\
\hline AGR-1423 & $19.7 \%$ & $18.9 \%$ & $18.62 \%$ \\
\hline AGR-1413 & $19.6 \%$ & $19.2 \%$ & $19.30 \%$ \\
\hline AGR-1442 & $16.9 \%$ & $16.5 \%$ & $16.59 \%$ \\
\hline AGR-1432 & $16.6 \%$ & $16.1 \%$ & $16.24 \%$ \\
\hline AGR-1422 & $16.2 \%$ & $16.3 \%$ & $16.47 \%$ \\
\hline AGR-1412 & $16.7 \%$ & $17.4 \%$ & $17.25 \%$ \\
\hline AGR-1441 & $17.7 \%$ & $18.4 \%$ & $18.84 \%$ \\
\hline AGR-1431 & $17.1 \%$ & $18.0 \%$ & $18.50 \%$ \\
\hline AGR-1421 & $17.3 \%$ & $18.3 \%$ & $18.66 \%$ \\
\hline AGR-1411 & $18.3 \%$ & $19.8 \%$ & $19.26 \%$ \\
\hline
\end{tabular}

Table 12. Burnup values for AGR-1 compacts from Capsule 3

\begin{tabular}{|c|c|c|c|}
\hline Compact ID & $\begin{array}{c}\text { Burnup from } \\
\text { Measured Cs-137 } \\
\text { Activity (\% FIMA) }\end{array}$ & $\begin{array}{c}\text { Burnup from } \\
\text { Measured Cs-134/Cs- } \\
\text { 137 Ratio (\% FIMA) }\end{array}$ & $\begin{array}{c}\text { Burnup from Ref. 4 } \\
\text { (ECAR-958) (\% FIMA) }\end{array}$ \\
\hline AGR-1343 & $19.6 \%$ & $19.9 \%$ & $19.47 \%$ \\
\hline AGR-1333 & $19.0 \%$ & $19.4 \%$ & $19.05 \%$ \\
\hline AGR-1323 & $20.1 \%$ & $19.4 \%$ & $19.03 \%$ \\
\hline AGR-1313 & $19.6 \%$ & $20.0 \%$ & $19.41 \%$ \\
\hline AGR-1342 & $17.0 \%$ & $17.6 \%$ & $17.46 \%$ \\
\hline AGR-1332 & $16.2 \%$ & $16.8 \%$ & $16.87 \%$ \\
\hline AGR-1322 & $15.7 \%$ & $16.9 \%$ & $16.87 \%$ \\
\hline AGR-1312 & $16.0 \%$ & $17.7 \%$ & $17.47 \%$ \\
\hline AGR-1341 & $17.9 \%$ & $19.3 \%$ & $19.38 \%$ \\
\hline AGR-1331 & $18.5 \%$ & $18.8 \%$ & $19.00 \%$ \\
\hline AGR-1321 & $18.2 \%$ & $18.6 \%$ & $18.98 \%$ \\
\hline AGR-1311 & $18.1 \%$ & $19.4 \%$ & $19.31 \%$ \\
\hline
\end{tabular}


Rev. 4

Analysis of Individual Compact Fission Product Inventory and Burnup for the AGR-1 TRISO Experiment using Title: Gamma Spectrometry

Table 13. Burnup values for AGR-1 compacts from Capsule 2

\begin{tabular}{|c|c|c|c|}
\hline Compact ID & $\begin{array}{c}\text { Burnup from } \\
\text { Measured Cs-137 } \\
\text { Activity (\% FIMA) }\end{array}$ & $\begin{array}{c}\text { Burnup from } \\
\text { Measured Cs-134/Cs- } \\
\text { 137 Ratio (\% FIMA) }\end{array}$ & $\begin{array}{c}\text { Burnup from Ref. 4 } \\
\text { (ECAR-958) (\% FIMA) }\end{array}$ \\
\hline AGR-1243 & $19.1 \%$ & $19.3 \%$ & $19.06 \%$ \\
\hline AGR-1233 & $18.5 \%$ & $18.6 \%$ & $18.31 \%$ \\
\hline AGR-1223 & $18.1 \%$ & $18.1 \%$ & $18.12 \%$ \\
\hline AGR-1213 & $18.4 \%$ & $18.4 \%$ & $18.35 \%$ \\
\hline AGR-1242 & $16.1 \%$ & $16.9 \%$ & $17.05 \%$ \\
\hline AGR-1232 & $17.0 \%$ & $15.9 \%$ & $16.16 \%$ \\
\hline AGR-1222 & $15.5 \%$ & $15.5 \%$ & $15.89 \%$ \\
\hline AGR-1212 & $16.0 \%$ & $16.2 \%$ & $16.18 \%$ \\
\hline AGR-1241 & $18.4 \%$ & $18.6 \%$ & $18.95 \%$ \\
\hline AGR-1231 & $17.9 \%$ & $17.7 \%$ & $18.28 \%$ \\
\hline AGR-1221 & $17.7 \%$ & $17.4 \%$ & $18.11 \%$ \\
\hline AGR-1211 & $17.9 \%$ & $17.7 \%$ & $18.29 \%$ \\
\hline
\end{tabular}

Table 14. Burnup values for AGR-1 compacts from Capsule 1

\begin{tabular}{|c|c|c|c|}
\hline Compact ID & $\begin{array}{c}\text { Burnup from } \\
\text { Measured Cs-137 } \\
\text { Activity (\% FIMA) }\end{array}$ & $\begin{array}{c}\text { Burnup from } \\
\text { Measured Cs-134/Cs- } \\
\text { 137 Ratio (\% FIMA) }\end{array}$ & $\begin{array}{c}\text { Burnup from Ref. 4 } \\
\text { (ECAR-958) (\% FIMA) }\end{array}$ \\
\hline AGR-1 143 & $16.5 \%$ & $17.5 \%$ & $17.29 \%$ \\
\hline AGR-1 133 & $16.3 \%$ & $16.2 \%$ & $16.14 \%$ \\
\hline AGR-1123 & $15.8 \%$ & $15.6 \%$ & $15.46 \%$ \\
\hline AGR-1113 & $15.8 \%$ & $15.6 \%$ & $15.21 \%$ \\
\hline AGR-1142 & $15.0 \%$ & $15.5 \%$ & $14.83 \%$ \\
\hline AGR-1132 & $13.9 \%$ & $14.2 \%$ & $13.76 \%$ \\
\hline AGR-1122 & $13.2 \%$ & $13.6 \%$ & $13.27 \%$ \\
\hline AGR-1 112 & $13.3 \%$ & $13.7 \%$ & $13.11 \%$ \\
\hline AGR-1 141 & $16.7 \%$ & $16.7 \%$ & $17.12 \%$ \\
\hline AGR-1 131 & $15.9 \%$ & $15.6 \%$ & $15.98 \%$ \\
\hline AGR-1 121 & $15.2 \%$ & $15.0 \%$ & $15.26 \%$ \\
\hline AGR-1 111 & $15.2 \%$ & $15.0 \%$ & $15.05 \%$ \\
\hline
\end{tabular}


Analysis of Individual Compact Fission Product Inventory and Burnup for the AGR-1 TRISO Experiment using

Title: Gamma Spectrometry

The ratio method for burnup determination also allows for a finer examination of how burnup varies across a compact. The formula in Equation (8) can be applied to each PGS measurement of a compact to find the local burnup for that slice. The slice burnup data for all 6 capsules is shown in Fig. 7 through Fig. 12. In these figures, the data from individual compacts has an artificial spacing in between each compact to assist in identifying the compacts. Interference from background and scans where only a fraction of the collimator was filled with part of the fueled region of the TRISO compact also made identification of the exact start and end of compact difficult to find. A systematic culling criteria was applied to the data in Fig. 7 through Fig. 12 to eliminate spurious burnup estimates taken from the activity ratio. If the measured activity of Cs-137 or Cs-134 was 10 times lower than the average compact slice measured activity, that slice was not included in the burnup estimate plots. This procedure accounts for the differences in the number of burnup estimates (e.g., 8 vs. 9) given for different compacts.

The resulting burnup estimates show definite trends in the local burnup across a compact of nearly 2\% FIMA for compacts on the edge of a capsule. For example Compact 2-1-2 in Fig. 11 has a minimum estimated slice burnup of $15.7 \%$ FIMA and a maximum estimated slice burnup of $17.2 \%$ FIMA. The uncertainty on these burnup estimates is also estimated to be around $3.1 \%$ which is mostly due to counting statistics and the geometry correction. The measured burnup values in Fig. 7 through Fig. 12 are compared to the results of the detailed inventory calculations from Reference 4. These simulations were performed with two calculation cells per compact that split the compact axially into two equal parts. Neutronics and inventory was tracked over both calculation cells, so each compact has two sets of simulated burnup values per compact in Fig. 7 through Fig. 12. The trends present in the activity ratio derived burnup estimates closely match the trends present in the simulation. A notable dissimilarity occurs in the measured and simulated burnup values for Stacks 1 and 3 . In the simulations, Stacks 1 and 3 tend to have almost the exact same burnup for each calculation cell. However, for the measured burnup estimates Stacks 1 and 3 are different in some capsules. One notable exception occurs in Fig. 9 where the burnup estimate for compact 411 is higher than the burnup estimate for compact 413. This single point of disagreement might suggest that the two compacts were switched at some point during handling; however, great care and double verification was always used during handling. Additionally, the total Cs-137 activity of compact 411 is lower than the total Cs-137 activity of compact 413 which suggests that compact 413 has a higher burnup. Compact 411 has been deconsolidated for individual particle gamma counting (IMGA). The average burnup from IMGA using the Cs-134 / Cs-137 ratio is 19.8\% FIMA which matches the PGS activity ratio measurements. Both the apparent burnup differences in Stack 1 and 3, and the behavior of compacts 413 and 411 need further investigation to fully understand the measured data. All the slice burnup estimates for each capsule are plotted in Fig. 13 to provide a general view of burnup behavior across the test train. 
Analysis of Individual Compact Fission Product Inventory and Burnup for the AGR-1 TRISO Experiment using Title: Gamma Spectrometry

ECAR No.: 1682

ECAR Rev. No.: 3

Project File No.: 23843

Date: $05 / 21 / 2014$

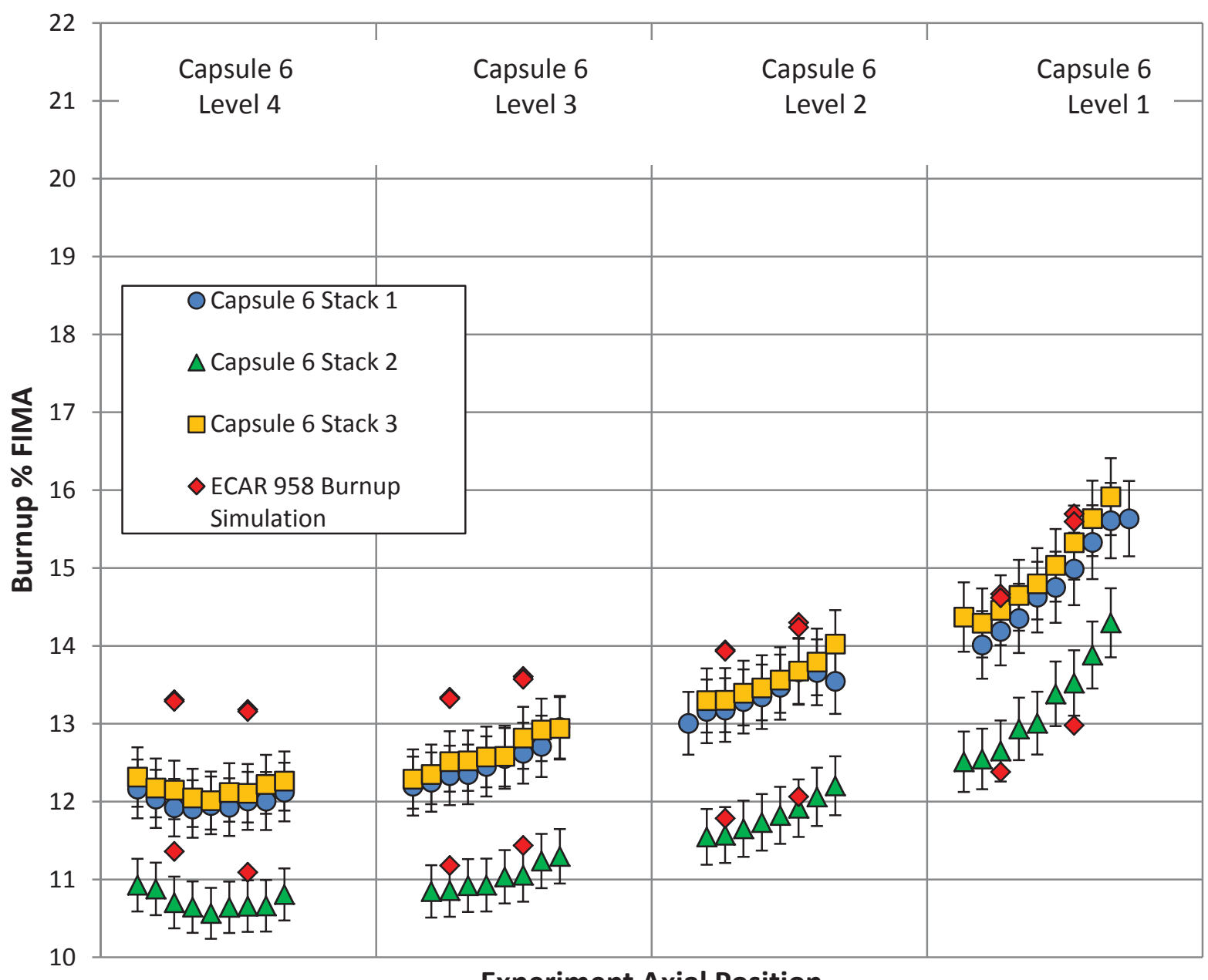

Experiment Axial Position

Fig. 7. Burnup derived from the Cs-134/Cs-137 activity ratio for each measured PGS slice for Capsule 6 compared to simulations from Reference 4 
Analysis of Individual Compact Fission Product Inventory and Burnup for the AGR-1 TRISO Experiment using Title: Gamma Spectrometry

ECAR No.: 1682

ECAR Rev. No.: 3

Project File No.: 23843

Date: $05 / 21 / 2014$

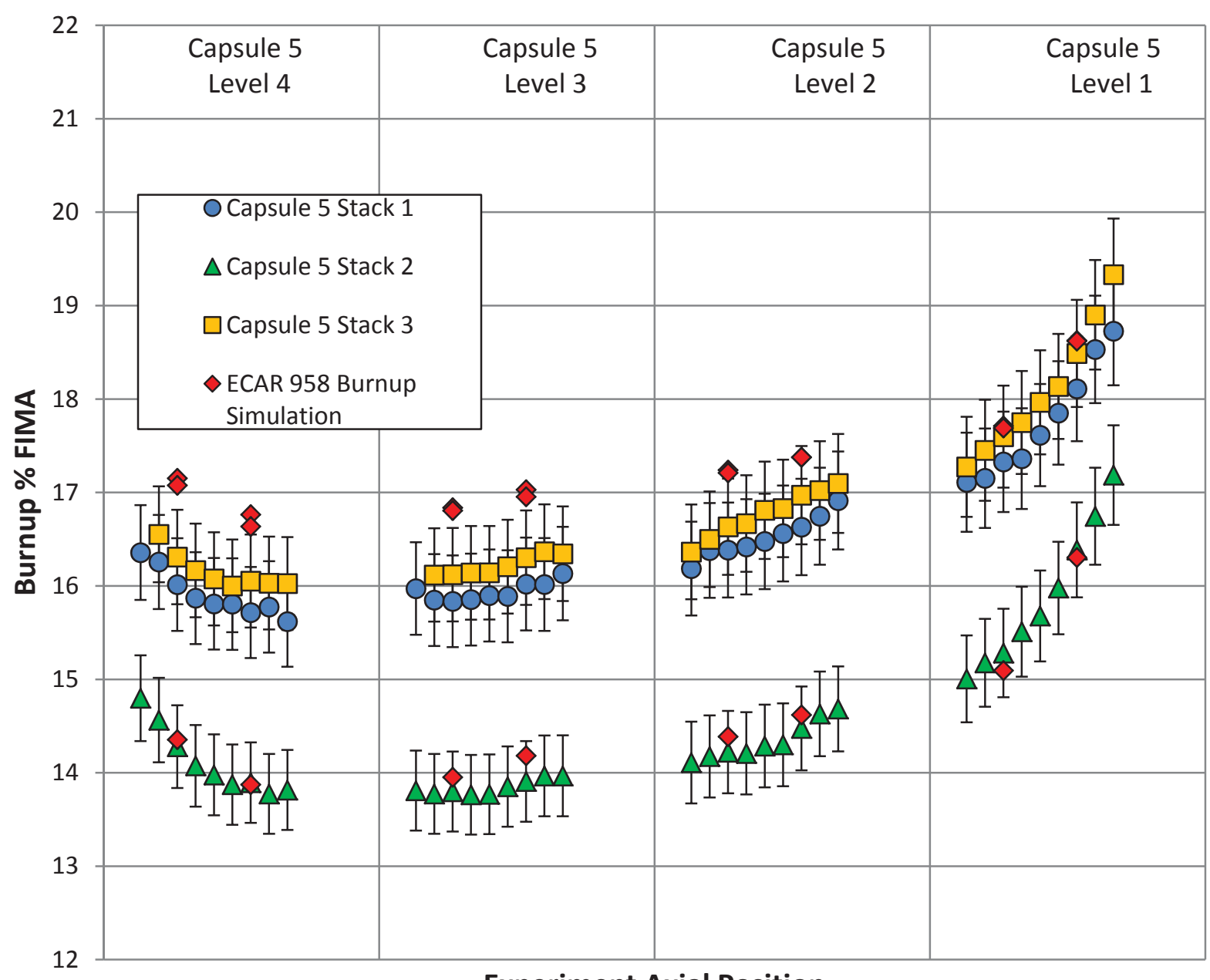

Experiment Axial Position

Fig. 8. Burnup derived from the Cs-134/Cs-137 activity ratio for each measured PGS slice for Capsule 5 compared to simulations from Reference 4 
Rev. 4

Analysis of Individual Compact Fission Product Inventory and Burnup for the AGR-1 TRISO Experiment using Title: Gamma Spectrometry

ECAR No.: 1682

ECAR Rev. No.: 3

Project File No.: 23843

Date: $05 / 21 / 2014$

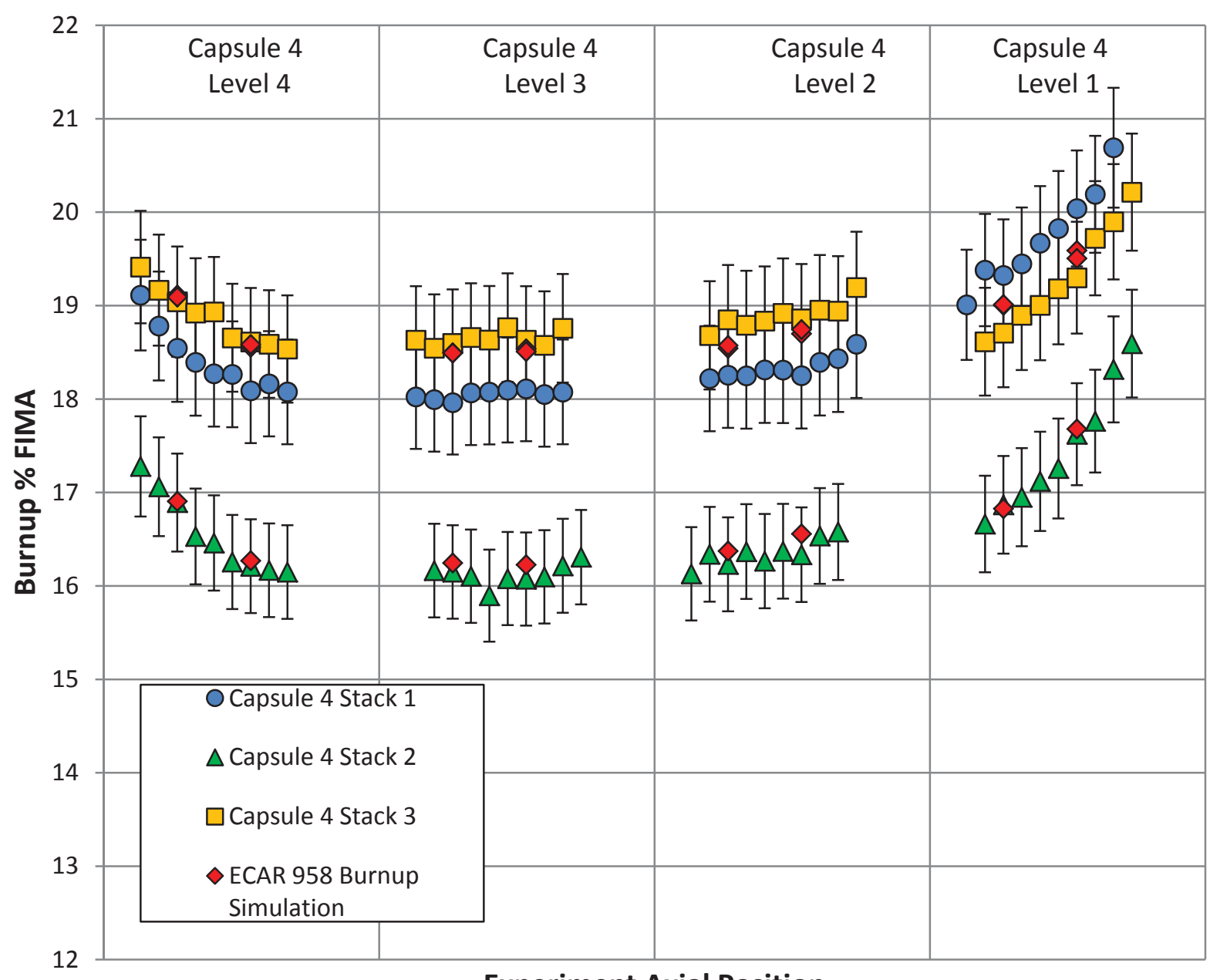

Experiment Axial Position

Fig. 9. Burnup derived from the Cs-134/Cs-137 activity ratio for each measured PGS slice for Capsule 4 compared to simulations from Reference 4 
Rev. 4

Analysis of Individual Compact Fission Product Inventory and Burnup for the AGR-1 TRISO Experiment using Title: Gamma Spectrometry

ECAR No.: 1682

ECAR Rev. No.: 3

Project File No.: 23843

Date: $05 / 21 / 2014$

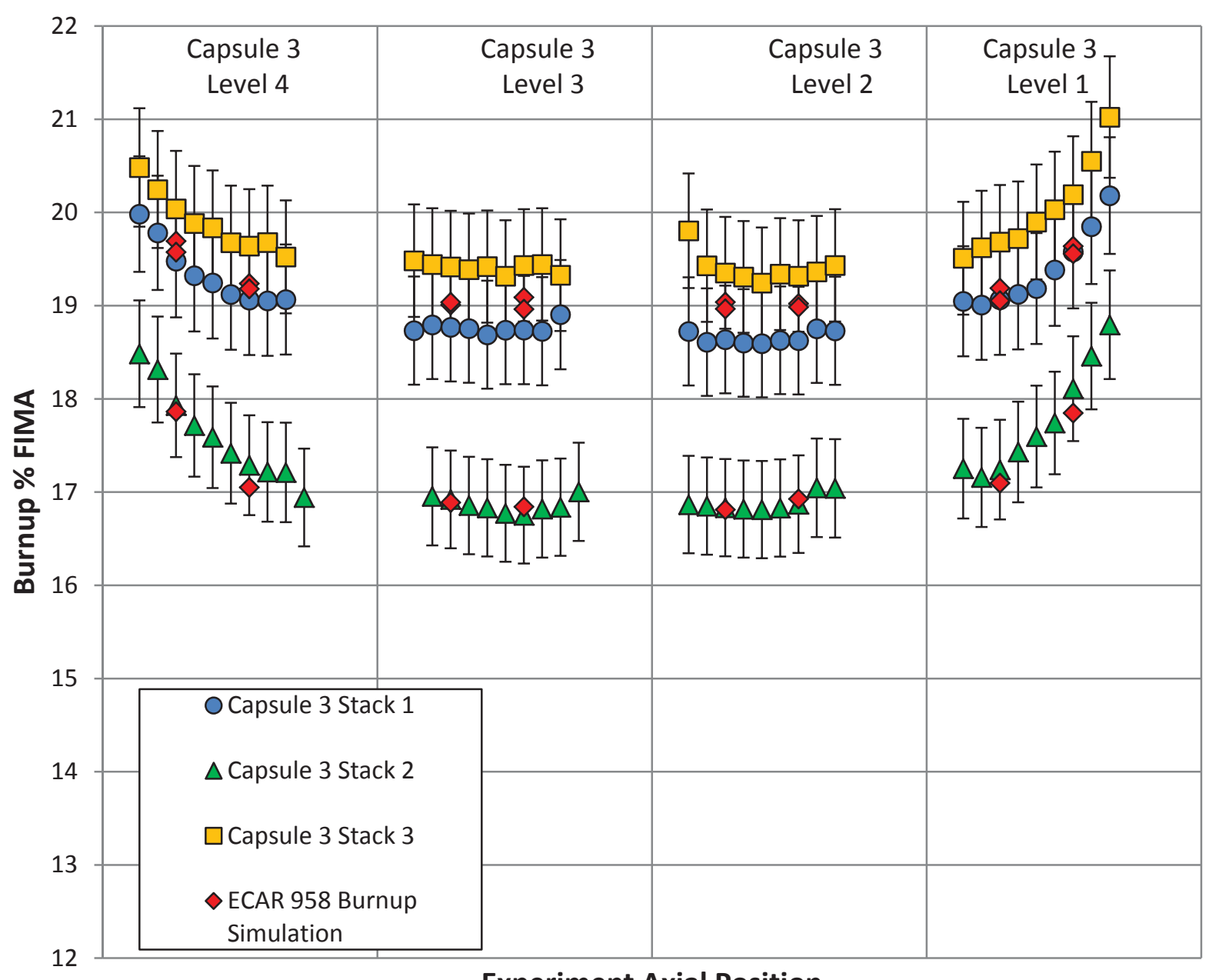

Experiment Axial Position

Fig. 10. Burnup derived from the Cs-134/Cs-137 activity ratio for each measured PGS slice for Capsule 3 compared to simulations from Reference 4 
Rev. 4

Analysis of Individual Compact Fission Product Inventory and Burnup for the AGR-1 TRISO Experiment using Title: Gamma Spectrometry

ECAR No.: 1682

ECAR Rev. No.: 3

Project File No.: 23843

Date: $05 / 21 / 2014$

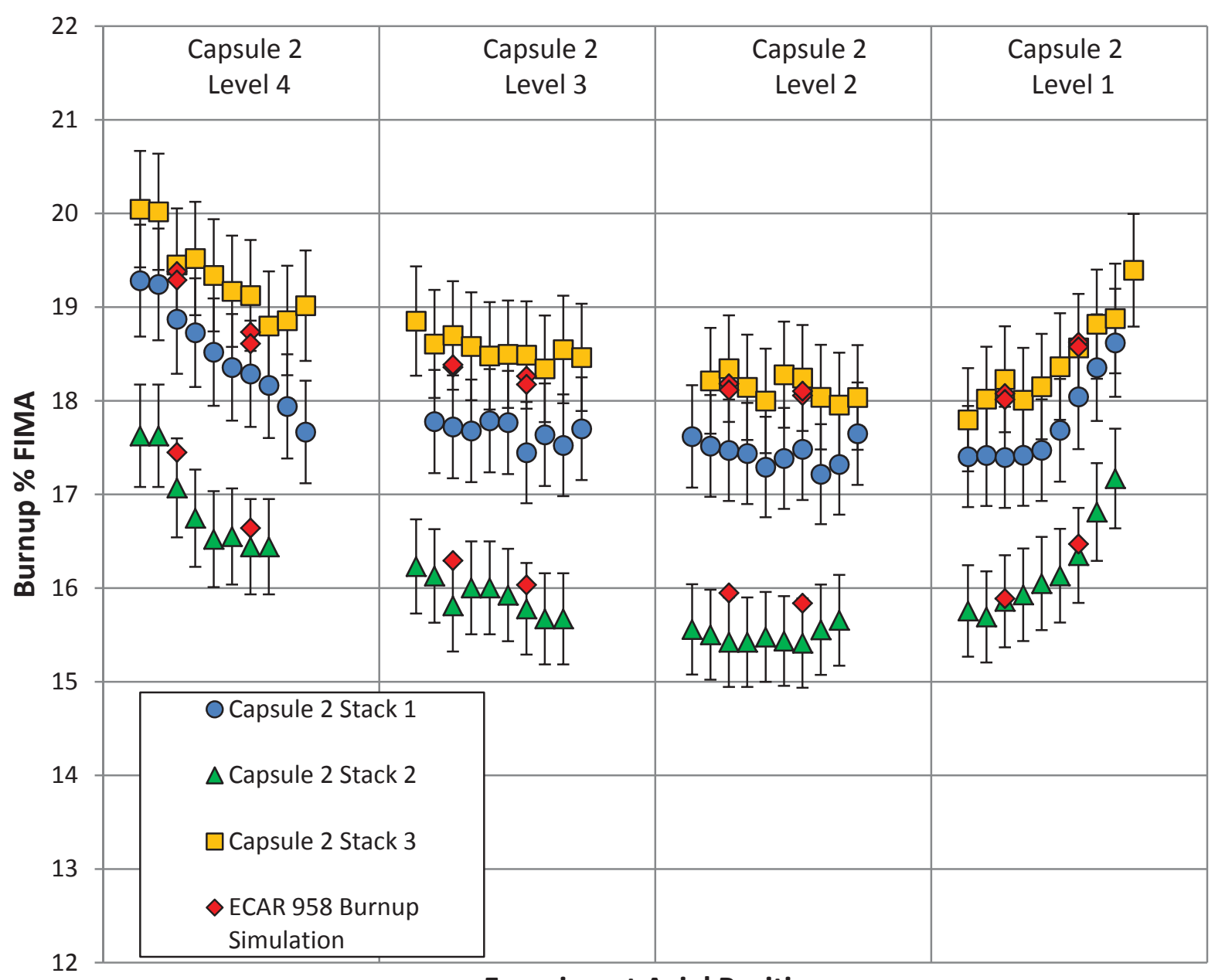

Experiment Axial Position

Fig. 11. Burnup derived from the Cs-134/Cs-137 activity ratio for each measured PGS slice for Capsule 2 compared to simulations from Reference 4 
Analysis of Individual Compact Fission Product Inventory and Burnup for the AGR-1 TRISO Experiment using Title: Gamma Spectrometry

ECAR No.: 1682

ECAR Rev. No.: 3

Project File No.: 23843

Date: $05 / 21 / 2014$

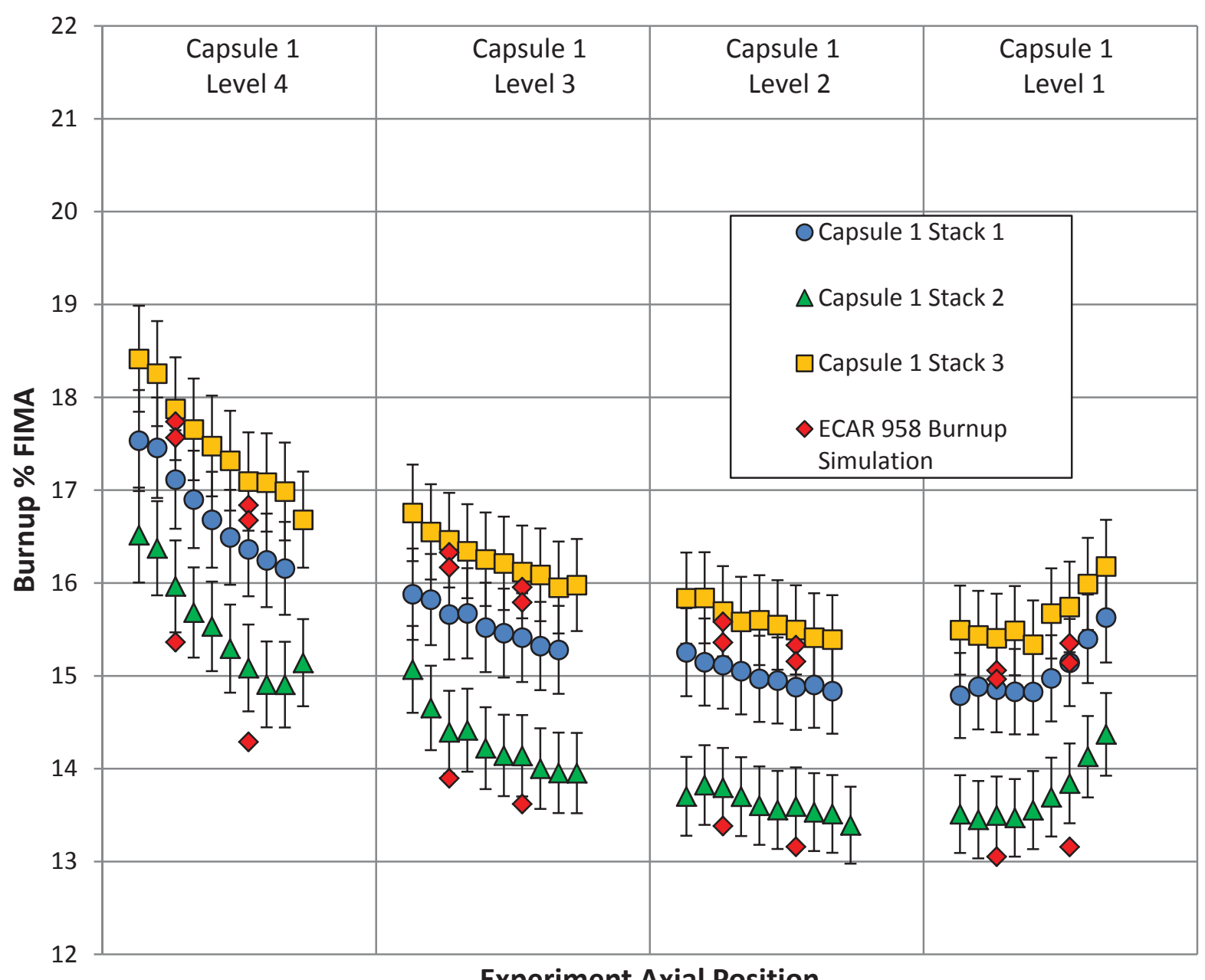

Experiment Axial Position

Fig. 12. Burnup derived from the Cs-134/Cs-137 activity ratio for each measured PGS slice for Capsule 1 compared to simulations from Reference 4 


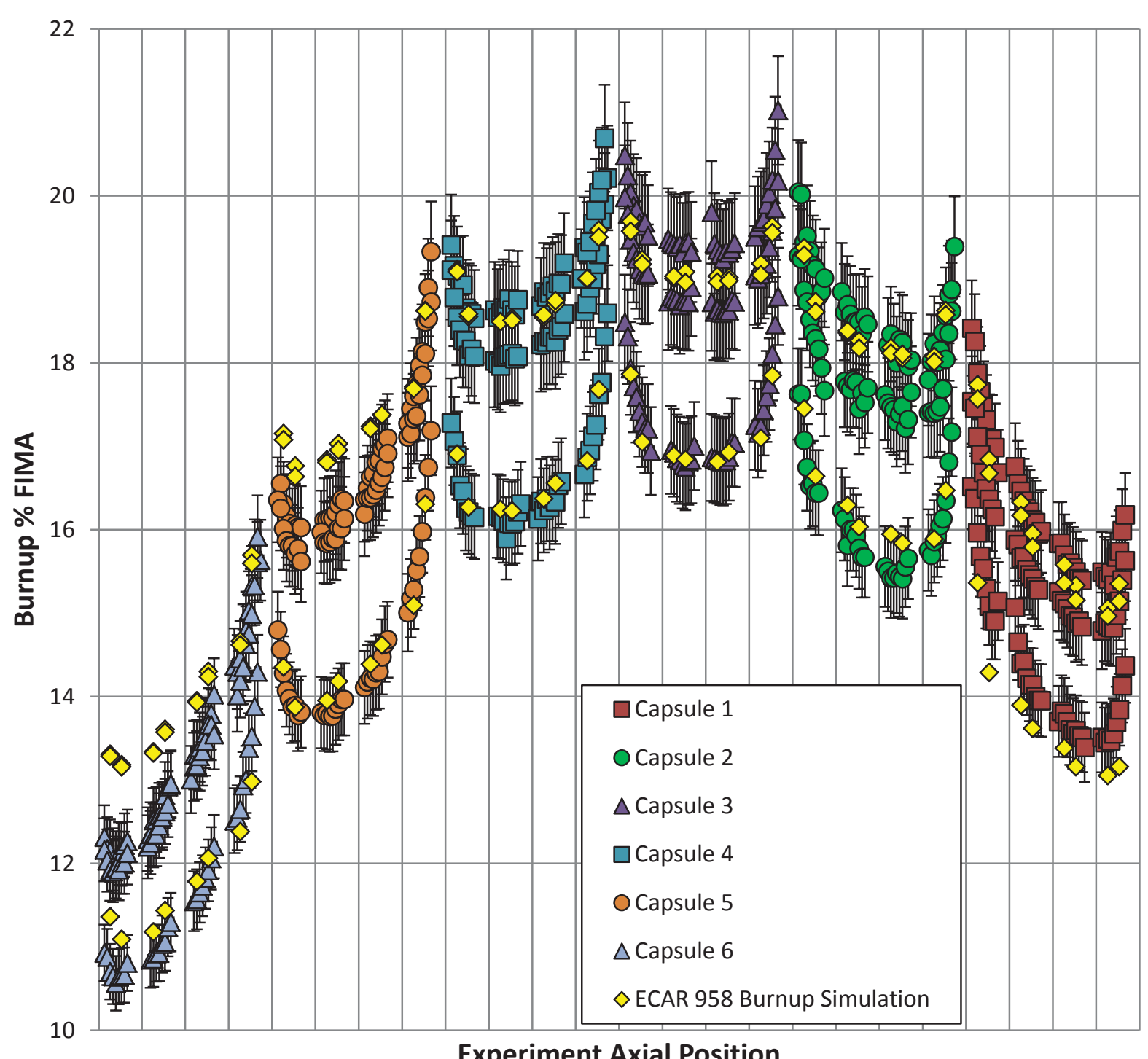

Fig. 13. Burnup derived from the Cs-134 / Cs-137 activity ratio for each measured PGS slice for all Capsules compared to simulations from ECAR-958. Each vertical tick mark represents a separate level in each capsule.

\subsection{Compact Rescanning for Ag-110m}

Due to the potential for significant silver release in-pile, quantification of the total Ag-110m inventory in the compacts at the end of irradiation is of critical importance for assessing fuel performance. These values are also important for interpreting subsequent high temperature safety test data, since some compacts may have released significant silver prior to safety testing. The initial scans were too rapid (10 minutes per scan) to capture the relatively weak Ag-110m signal below the Cs-137 signal. The $661.62 \mathrm{keV} \mathrm{Cs}-137$ gamma obscured the primary Ag-110m gamma at $657 \mathrm{keV}$. In order to evaluate Ag-110m, it was necessary to scan for longer periods of time to allow the lower yield peaks of Ag-110m at 884 and $937 \mathrm{keV}$ to become quantifiable. Initial attempts at counting the compacts for longer times to quantify Ag-110m were marginally successful. An upgrade of the PGS HPGe detector and the addition of a Compton suppression system helped cut down the time from 8 hours per scan to 3 hours per scan to collect adequate data. 
Analysis of Individual Compact Fission Product Inventory and Burnup for the AGR-1 TRISO Experiment using Title: Gamma Spectrometry

All of the available 56 (of 72) AGR-1 compacts were rescanned on the updated PGS system to determine Ag-110m inventory. Scanning parameters were similar to those described above, with the exception of a longer counting duration (3 hours) for each slice. Several of compacts had been destructively examined prior to undertaking these measurements and therefore were not available. The total compact Ag-110m inventory was calculated from the individual scans in the same manner as described above for the other isotopes of interest. The results of these measurements are displayed in Table 15 and Table 16, and the data is visually summarized in Fig. 14. The results of this study revealed that the Ag- $110 \mathrm{~m}$ present in the compacts was often very different from the inventory predicted in ECAR-958, indicating very different Ag-110m release magnitudes between the compacts. Some of the trends in the data are illustrated by Fig. 14. Ag-110m retention tended to be higher at the top and bottom of capsules (Levels 4 and 1) especially in Capsules 1 and 5. This may be indicative of a thermally driven release mechanism, as the average compact temperatures were lower at the capsule ends than in the capsule centers. It should be noted that these measurements evaluated Ag-110m in both the particles and in the compact matrix, so by these measurements alone it cannot be ruled out that the higher Ag- $110 \mathrm{~m}$ fractions at the end of the capsule stacks are not the result of migration from the inner compacts. However, available data from deconsolidation-leach-burn-leach of the AGR-1 compacts indicates that the amount of Ag-110m in the compact matrix is generally low relative to the amount of release indicated in Fig 14 (all but one compact had less than 3.5\% of the total Ag-110m inventory in the matrix) ${ }^{15}$, suggesting that migration from one compact to another is not a significant contributor to total Ag-110m inventory.

Compacts at the same Level in Stacks 1 and Stack 3 have very similar Ag-110m retention fractions. This behavior is consistent enough across the whole test train that if one compact from Stack 1 or 3 at a particular level was not gamma scanned, its retention fraction can be inferred from the scanned compact. The retention fraction in Capsule 3 was often above 1.0 , indicating very little loss from the compacts and probably some bias in the simulations. Subsequent measurements of the Capsule 3 holder and other capsule components showed that most of the Ag-110m produced in these compacts was retained compared to the other compacts (only $1.2 \%$ of the total capsule inventory of Ag- $110 \mathrm{~m}$ was found outside of the compacts) ${ }^{16}$. Capsule 6 was the hottest capsule in the AGR-1 experiment and also lost the most Ag-110m from the compacts, again consistent with the analysis of the capsule components, which showed that Capsule 6 had the highest fraction of Ag-110m outside of the compacts (38\%). The retention fraction for Compact 6-2-3 was only 0.0828, indicating almost complete release of silver from this compact which had the highest calculated time-average volume-average (TAVA) temperature $\left(1137^{\circ} \mathrm{C}\right)$. There is a loose inverse correlation between TAVA temperature and Ag- $110 \mathrm{~m}$ retention fraction, but the there may be a threshold temperature before Ag-110m migration. Ongoing fuel performance modeling work has indicated that TAVA temperatures may be inadequate for investigating and predicting silver behavior, as temperature distributions within the compacts could be large in some cases and temperatures could vary widely throughout the irradiation. A more in-depth analysis of silver retention in the compacts, which takes into account the complex temperature histories of the particles, is in progress. 
Rev. 4

Analysis of Individual Compact Fission Product Inventory and Burnup for the AGR-1 TRISO Experiment using Title: Gamma Spectrometry

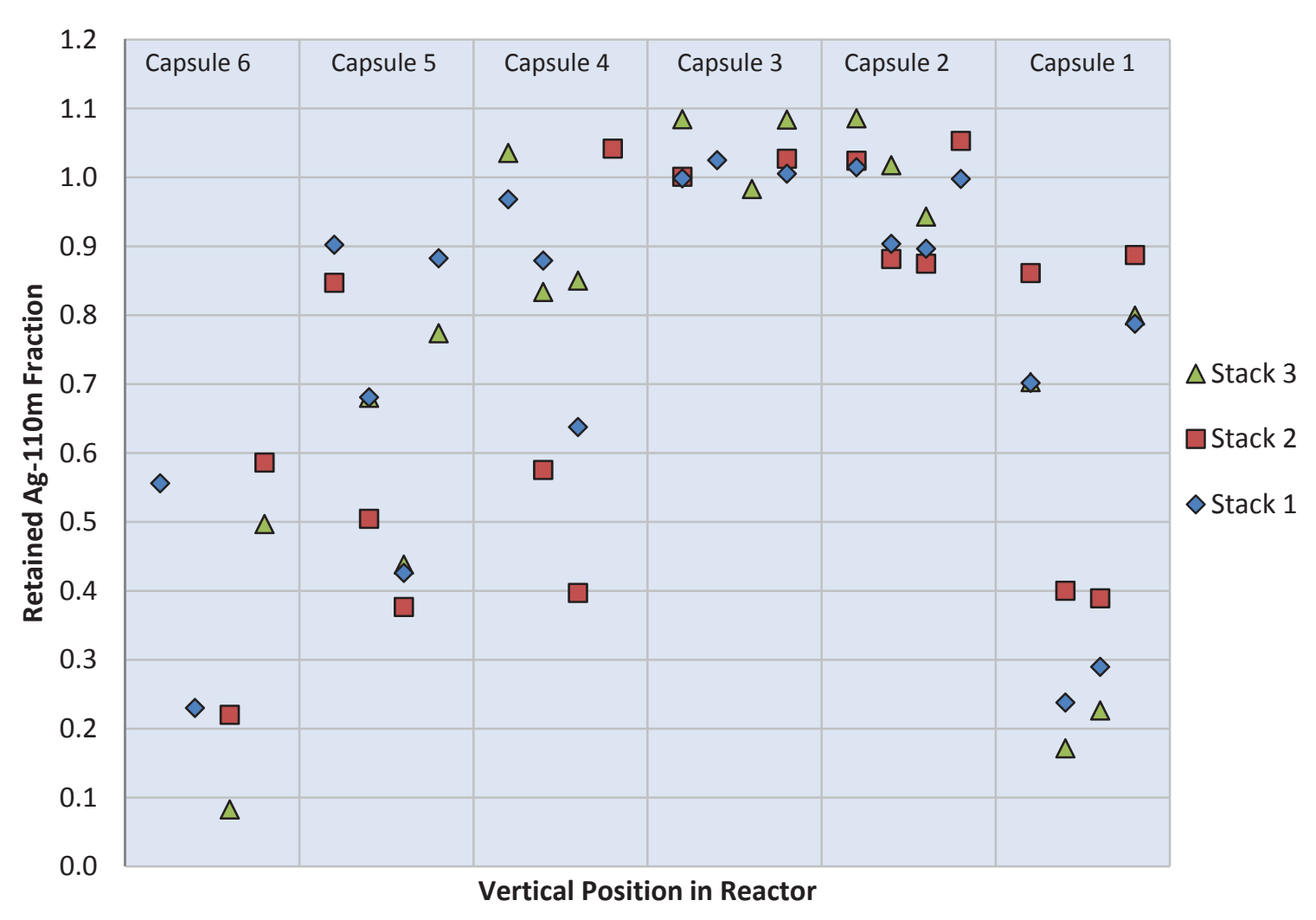

Fig. 14. Retained Ag-110m fraction for AGR-1 compacts. 
Rev. 4

Analysis of Individual Compact Fission Product Inventory and Burnup for the AGR-1 TRISO Experiment using

Title: Gamma Spectrometry

ECAR No.: 1682

ECAR Rev. No.: 3

Project File No.: 23843

Date: $05 / 21 / 2014$

Table 15. Amount of Ag-110m measured in available compacts along with the retained fraction, burnup, and TAVA temperature for Capsules 6, 5, and 4

\begin{tabular}{|c|c|c|c|c|}
\hline Compact & $\begin{array}{l}\text { Ag-110m Decay } \\
\text { Corrected } \\
\text { Activity }(\mu \mathrm{Ci})\end{array}$ & $\begin{array}{c}\text { Ratio of Measured } \\
\text { to Simulated } \\
\text { Activity }\end{array}$ & $\begin{array}{c}\text { Burnup based on } \\
\text { Cs-134 to Cs-137 } \\
\text { Ratio (\% FIMA) }\end{array}$ & $\begin{array}{c}\text { Time Average Volume } \\
\text { Average Temperatures } \\
\text { of Compact }\left({ }^{\circ} \mathrm{C}\right)\end{array}$ \\
\hline AGR-1 6-4-3 & N/A & N/A & 12.14 & 1042 \\
\hline AGR-1 6-3-3 & $\mathrm{N} / \mathrm{A}$ & $\mathrm{N} / \mathrm{A}$ & 12.62 & 1106 \\
\hline AGR-16-2-3 & 252.22 & 0.0828 & 13.57 & 1137 \\
\hline AGR-1 6-1-3 & 1859.79 & 0.4970 & 14.95 & 1112 \\
\hline AGR-1 6-4-2 & $\mathrm{N} / \mathrm{A}$ & $\mathrm{N} / \mathrm{A}$ & 10.71 & 1009 \\
\hline AGR-1 6-3-2 & $\mathrm{N} / \mathrm{A}$ & $\mathrm{N} / \mathrm{A}$ & 11.03 & 1070 \\
\hline AGR-16-2-2 & 443.24 & 0.2203 & 11.82 & 1101 \\
\hline AGR-1 6-1-2 & 1481.56 & 0.5863 & 13.25 & 1080 \\
\hline AGR-1 6-4-1 & 1210.00 & 0.5563 & 11.99 & 1041 \\
\hline AGR-1 6-3-1 & 574.72 & 0.2301 & 12.45 & 1106 \\
\hline AGR-1 6-2-1 & N/A & $\mathrm{N} / \mathrm{A}$ & 13.36 & 1136 \\
\hline AGR-1 6-1-1 & $\mathrm{N} / \mathrm{A}$ & $\mathrm{N} / \mathrm{A}$ & 14.77 & 1112 \\
\hline AGR-1 5-4-3 & $\mathrm{N} / \mathrm{A}$ & $\mathrm{N} / \mathrm{A}$ & 16.16 & 1000 \\
\hline AGR-1 5-3-3 & 3600.63 & 0.6803 & 16.21 & 1042 \\
\hline AGR-1 5-2-3 & 2502.14 & 0.4381 & 16.79 & 1060 \\
\hline AGR-1 5-1-3 & 5042.57 & 0.7739 & 18.04 & 1043 \\
\hline AGR-1 5-4-2 & 2968.46 & 0.8472 & 14.07 & 963 \\
\hline AGR-1 5-3-2 & 1809.15 & 0.5047 & 13.85 & 1002 \\
\hline AGR-1 5-2-2 & 1469.26 & 0.3767 & 14.35 & 1021 \\
\hline AGR-1 5-1-2 & $\mathrm{N} / \mathrm{A}$ & $\mathrm{N} / \mathrm{A}$ & 15.79 & 1008 \\
\hline AGR-1 5-4-1 & 4552.48 & 0.9023 & 15.91 & 998 \\
\hline AGR-1 5-3-1 & 3538.04 & 0.6809 & 15.93 & 1041 \\
\hline AGR-1 5-2-1 & 2401.21 & 0.4263 & 16.53 & 1058 \\
\hline AGR-1 5-1-1 & 5660.88 & 0.8828 & 17.73 & 1041 \\
\hline AGR-14-4-3 & 7779.61 & 1.0356 & 18.85 & 1060 \\
\hline AGR-14-3-3 & 6147.66 & 0.8339 & 18.65 & 1095 \\
\hline AGR-14-2-3 & 6456.02 & 0.8504 & 18.87 & 1101 \\
\hline AGR-14-1-3 & $\mathrm{N} / \mathrm{A}$ & $\mathrm{N} / \mathrm{A}$ & 19.22 & 1076 \\
\hline AGR-1 4-4-2 & $\mathrm{N} / \mathrm{A}$ & $\mathrm{N} / \mathrm{A}$ & 16.53 & 1025 \\
\hline AGR-1 4-3-2 & 2941.60 & 0.5756 & 16.10 & 1057 \\
\hline AGR-1 4-2-2 & 2116.45 & 0.3972 & 16.34 & 1065 \\
\hline AGR-1 4-1-2 & 6133.63 & 1.0418 & 17.37 & 1043 \\
\hline AGR-1 4-4-1 & 7122.56 & 0.9682 & 18.38 & 1058 \\
\hline AGR-1 4-3-1 & 6319.41 & 0.8794 & 18.05 & 1092 \\
\hline AGR-1 4-2-1 & 4748.33 & 0.6377 & 18.31 & 1099 \\
\hline AGR-1 4-1-1 & $\mathrm{N} / \mathrm{A}$ & $\mathrm{N} / \mathrm{A}$ & 19.80 & 1073 \\
\hline
\end{tabular}


Rev. 4

Analysis of Individual Compact Fission Product Inventory and Burnup for the AGR-1 TRISO Experiment using

Title: Gamma Spectrometry
ECAR No.: 1682
ECAR Rev. No.: 3
Project File No.: 23843
Date: $05 / 21 / 2014$

Table 16. Amount of Ag-110m measured in available compacts along with the retained fraction, burnup, and TAVA temperature for Capsules 3, 2, and 1

\begin{tabular}{|c|c|c|c|c|}
\hline Compact & $\begin{array}{l}\text { Ag-110m Decay } \\
\text { Corrected } \\
\text { Activity }(\mu \mathrm{Ci}) \\
\end{array}$ & $\begin{array}{c}\text { Ratio of Measured } \\
\text { to Simulated } \\
\text { Activity }\end{array}$ & $\begin{array}{c}\text { Burnup based on } \\
\text { Cs-134 to Cs-137 } \\
\text { Ratio (\% FIMA) }\end{array}$ & $\begin{array}{c}\text { Time Average Volume } \\
\text { Average Temperatures } \\
\text { of Compact }\left({ }^{\circ} \mathrm{C}\right)\end{array}$ \\
\hline AGR-1 3-4-3 & 9336.10 & 1.0845 & 19.92 & 1029 \\
\hline AGR-1 3-3-3 & $\mathrm{N} / \mathrm{A}$ & $\mathrm{N} / \mathrm{A}$ & 19.41 & 1054 \\
\hline AGR-13-2-3 & 8040.97 & 0.9832 & 19.35 & 1054 \\
\hline AGR-1 3-1-3 & 9238.33 & 1.0839 & 19.98 & 1025 \\
\hline AGR-1 3-4-2 & 6118.61 & 1.0010 & 17.62 & 997 \\
\hline AGR-1 3-3-2 & $\mathrm{N} / \mathrm{A}$ & $\mathrm{N} / \mathrm{A}$ & 16.85 & 1020 \\
\hline AGR-13-2-2 & $\mathrm{N} / \mathrm{A}$ & $\mathrm{N} / \mathrm{A}$ & 16.87 & 1020 \\
\hline AGR-1 3-1-2 & 6250.57 & 1.0272 & 17.75 & 994 \\
\hline AGR-1 3-4-1 & 8332.63 & 0.9987 & 19.34 & 1026 \\
\hline AGR-1 3-3-1 & 8145.11 & 1.0251 & 18.75 & 1052 \\
\hline AGR-1 3-2-1 & $N / A$ & $\mathrm{~N} / \mathrm{A}$ & 18.65 & 1051 \\
\hline AGR-1 3-1-1 & 8367.72 & 1.0053 & 19.43 & 1023 \\
\hline AGR-1 2-4-3 & 8368.77 & 1.0859 & 19.32 & 1016 \\
\hline AGR-1 2-3-3 & 7130.49 & 1.0178 & 18.55 & 1033 \\
\hline AGR-1 2-2-3 & 6311.84 & 0.9432 & 18.15 & 1023 \\
\hline AGR-1 2-1-3 & $N / A$ & $\mathrm{~N} / \mathrm{A}$ & 18.42 & 988 \\
\hline AGR-1 2-4-2 & 5679.92 & 1.0244 & 16.88 & 985 \\
\hline AGR-1 2-3-2 & 4360.09 & 0.8818 & 15.91 & 998 \\
\hline AGR-1 2-2-2 & 4102.35 & 0.8749 & 15.48 & 987 \\
\hline AGR-1 2-1-2 & 4980.39 & 1.0530 & 16.20 & 955 \\
\hline AGR-1 2-4-1 & 7607.36 & 1.0148 & 18.57 & 1013 \\
\hline AGR-1 2-3-1 & 6149.99 & 0.9037 & 17.67 & 1030 \\
\hline AGR-1 2-2-1 & 5864.60 & 0.8967 & 17.40 & 1020 \\
\hline AGR-1 2-1-1 & 6487.39 & 0.9978 & 17.75 & 986 \\
\hline AGR-1 1-4-3 & 3668.82 & 0.7030 & 17.49 & 1073 \\
\hline AGR-1 1-3-3 & 755.20 & 0.1717 & 16.25 & 1094 \\
\hline AGR-1 1-2-3 & 878.54 & 0.2266 & 15.58 & 1073 \\
\hline AGR-1 1-1-3 & 2843.18 & 0.7995 & 15.62 & 1019 \\
\hline AGR-1 1-4-2 & 3185.19 & 0.8612 & 15.52 & 1046 \\
\hline AGR-1 1-3-2 & 1232.96 & 0.4002 & 14.24 & 1065 \\
\hline AGR-1 1-2-2 & 1042.79 & 0.3894 & 13.64 & 1042 \\
\hline AGR-1 1-1-2 & 2163.88 & 0.8876 & 13.72 & 990 \\
\hline AGR-1 1-4-1 & 3547.30 & 0.7019 & 16.73 & 1072 \\
\hline AGR-1 1-3-1 & 1015.71 & 0.2380 & 15.58 & 1093 \\
\hline AGR-1 1-2-1 & 1087.52 & 0.2899 & 15.00 & 1071 \\
\hline AGR-1 1-1-1 & 2722.55 & 0.7877 & 15.03 & 1018 \\
\hline
\end{tabular}


Rev. 4

Analysis of Individual Compact Fission Product Inventory and Burnup for the AGR-1 TRISO Experiment using Title: Gamma Spectrometry

\subsection{Conclusions}

Gamma-ray spectrometry scans of irradiated TRISO fuel from the AGR-1 experiment have recently been completed using the Precision Gamma Scanning system located at the HFEF facility of the INL MFC complex. Fuel compact burnup values derived from Cs-137 activity measurements and the activity ratio of Cs-134 to Cs-137 agree quite well with the expected AGR-1 burnup calculated by coupled Monte Carlo transport and depletion simulations. On-going work will allow corroboration of burnup analysis by gamma-ray spectrometry using the PGS with individual TRISO particle gamma scans, chemical burnup analysis and simulation.

\section{REFERENCES}

1. M.A. Pope, “AGR-1 Irradiation Test Final As-Run Report,” INL/EXT-10-18097, Idaho National Laboratory, (2010)

2. S.T. Hsue, T.W. Crane, et al, "Nondestructive Assay Methods for Irradiated Nuclear Fuels," LA-6923, Los Alamos Scientific Laboratory, (1978)

3. P.L. Winston, J.W. Sterbentz, "Gross Gamma Dose Rate Measurements for TRIGA spent Nuclear Fuel Burnup Validation," Proc. ICONE10, Arlington, VA, (2002)

4. J.W. Sterbentz, "JMOCUP As-Run Daily Depletion Calculations for the AGR-1 Experiment in ATR B-10 Position," ECAR-958 Rev. 2, Idaho National Laboratory, (2011)

5. Idaho National Laboratory Operating Instructions, "Precision Gamma Scanner" HFEF-OI-4352, (2010)

6. R.P. Lind, "AGR-1 Gamma Scan Data to date," Data Transmittal 11PL007, NGNP project files INL EDMS, Idaho National Laboratory, (2011)

7. INL Drawing 761964, "MFC 785 AGR - 1 PGS COMPACT CONTAINER ASSEMBLY," (2010)

8. INL Drawing 761960, "MFC 785 AGR - 1 PGS COMPACT STACK TUBE W / COMPACTS ASSEMBLY," (2010)

9. M.W. Drigert, "Determination of absolute activities of ${ }^{152} \mathrm{Eu}$ sources used by the PGS for AGR-1 PIE," ECAR-991, Idaho National Laboratory, (2010)

10. INL Drawing 766032, "MFC 785 NGNP AGR-1 COMPACT PGS SINGLE CALIBRATION SOURCE HOLDER ASSEMBLY,” (2010)

11. INL Drawing 766031, "MFC 785 NGNP AGR-1 COMPACT PGS DOUBLE CALIBRATION SOURCE HOLDER ASSEMBLY," (2010)

12. P. A. Demkowicz, “AGR-1 Post-Irradiation Examination Plan,” PLN-2828 Rev. 1, Idaho National Laboratory, (2010)

13. J.T. Maki, "AGR-1 Irradiation Experiment Test Plan,” INL/EXT-05-00593 Rev. 3, Idaho National Laboratory, (2009)

14. D. Freis, E.R. Nasyrow, H. Toscano, "BURN-UP DETERMINATION OF HIGH TEMPERATURE REACTOR SPHERICALFUEL ELEMENTS BY GAMMA SPECTROMETRY," Proc. HTR2006, Johannesburg, South Africa (2006)

15. P.A. Demkowicz, J.D. Hunn, R.N. Morris, J.M. Harp, P.L. Winston, C.A. Baldwin, F.C. Montgomery, S.A. Ploger, "Prelimnary Results of Post-Irradiation Examination of the AGR-1 TRISO Fuel Compacts, HTR-2012-3-021, Proceedings of HTR-2012, Tokyo, Japan (2012)

16. P.A. Demkowicz, J.M. Harp, P.L. Winston, S.A. Ploger, "Analysis of Fission Products on the AGR-1 Capsule Components," INL/EXT-13-28483, Idaho National Laboratory, (2013) 
Rev. 4

Analysis of Individual Compact Fission Product Inventory and Burnup for the AGR-1 TRISO Experiment using Title: Gamma Spectrometry

\section{APPENDIXES}

\subsection{Appendix A - Example PC-GAP Listing (.LIS) File}

PC-GAP analyzes spectrum files and produces a Listing file (.LIS) that contains the activity of all identified nuclides in the analyzed spectrum as well as all the identified peaks and peak areas. To identify peaks and calculate accurate activities PC-GAP must be supplied with the appropriate energy calibration and efficiency curve. Below is a sample from an example .LIS file from a scan of Compact 4-4-3. The collected spectrum was GS0603101525001042.CHN. 
Rev. 4

Analysis of Individual Compact Fission Product Inventory and Burnup for the AGR-1 TRISO Experiment using

Title: Gamma Spectrometry

ECAR No.: 1682

ECAR Rev. No.: 3

Project File No.: 23843

Date: $05 / 21 / 2014$

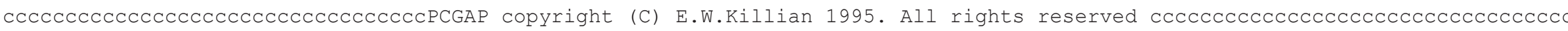

\section{$21-M A R-11 \quad 13: 38$}

PULSE-HEIGHT SPECTRAL ANALYSIS

* SAMPLE NAME: ORTEC SPECTRUM C: \RESEARCH \AGR-PIE-GAMMASCAN \SPECTRA \AGR1-CAPSULES13456-CHN $\backslash 06031$

SAMPLE NUMBER: $\quad 1$

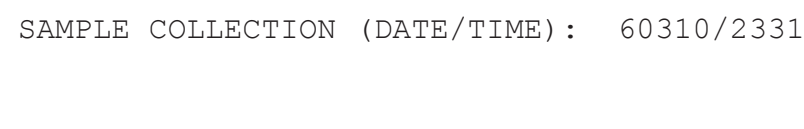

* EFFiCIEnCy TABLE: GS00006226 .... Compact Slice Efficiency 
Rev. 4

Analysis of Individual Compact Fission Product Inventory and Burnup for the AGR-1 TRISO Experiment using

Title: Gamma Spectrometry

ECAR No.: $1682 \quad$ ECAR Rev. No.: $3 \quad$ Project File No.: $23843 \quad$ Date: 05/21/2014

--BACKGROUNDS AVERAGED--

Widths marked with ** deviate from the width equation by more than $20 \%$

CENTROID WIDTH ENERGY AREA OERR CHI EFFICIENCY PEAK CPS COR CPS COR GAM/SEC

\begin{tabular}{|c|c|c|c|c|c|c|c|c|c|}
\hline 142.00 & 5.8 & 24.298 & 863. & 36.90 & 1.2 & $1.000 \mathrm{E}+00$ & $1.44 \mathrm{E}+00$ & $1.44 \mathrm{E}+00$ & $1.44 \mathrm{E}+00$ \\
\hline 451.74 & 7.3 & 82.186 & 45895 . & 1.04 & 1.2 & $6.010 \mathrm{E}-07$ & $7.65 E+01$ & $7.65 E+01$ & $1.27 \mathrm{E}+08$ \\
\hline
\end{tabular}

$\begin{array}{cccccc} & \text { TAG } & \text { FIT FLAG } & \text { BR }(\%) & \text { T }(1 / 2) \text { HR } & \text { DECAY CORR. INTENSITY CODE } \\ 66 \text { DY } 166 & 0 & 13.80 & 8.16 \mathrm{E}+01 & 1.001 \mathrm{E}+00 & 1\end{array}$

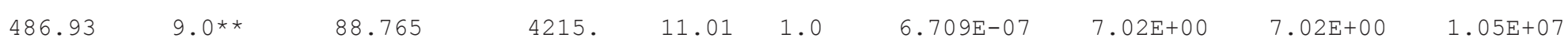

$\begin{array}{llrrrrc} & \text { TAG } & \text { FIT FLAG } & \text { BR }\left(\frac{\circ}{0}\right) & \text { T }(1 / 2) \mathrm{HR} & \text { DECAY CORR. INTENSITY CODE } \\ 48 \mathrm{CD} 109 & 0 & 3.65 & 1.11 \mathrm{E}+04 & 1.000 \mathrm{E}+00 & 1 \\ 46 \mathrm{PD} 109 & 0 & 3.61 & 1.37 \mathrm{E}+01 & 1.006 \mathrm{E}+00 & 1 \\ 63 \mathrm{EU} 156 & 0 & 8.87 & 3.65 \mathrm{E}+02 & 1.000 \mathrm{E}+00 & 2 \\ 72 \mathrm{HF} & 175 & 0 & 2.35 & 1.68 \mathrm{E}+03 & 1.000 \mathrm{E}+00 & 3\end{array}$


Rev. 4

Analysis of Individual Compact Fission Product Inventory and Burnup for the AGR-1 TRISO Experiment using

Title: Gamma Spectrometry

ECAR No.: 1682 ECAR Rev. No.: $3 \quad$ Project File No.: $23843 \quad$ Date: 05/21/2014

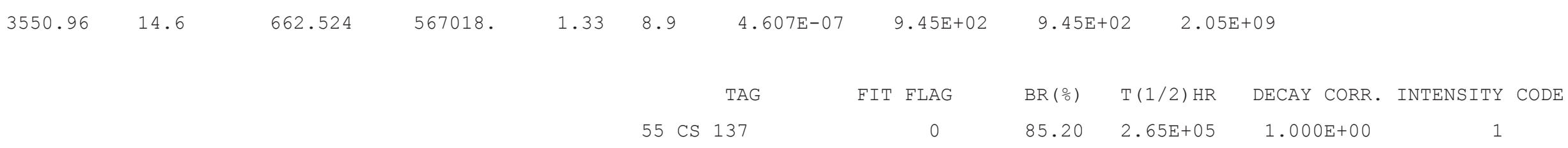

ISOTOPE SUMMARY

\begin{tabular}{|c|c|c|c|c|c|c|c|c|c|c|c|c|}
\hline & ISOTOPE & LIB ENG & LINE ENG & $I-C$ & FLAGS & OTHER TAGS & MICRO-CI/UNIT & ㅇRROR & NET & MICRO-CI/UNIT & $\mathrm{NET}$ & ERROR ( 1 -S IGMA \\
\hline & RURH 106 & 621.92 & 622.90 & 1 & 0 & & $1.385 \mathrm{E}+05+$ & 1.13 & & & & \\
\hline & & 1050.36 & 1050.93 & 2 & 0 & & $1.319 \mathrm{E}+05+$ & 2.21 & & & & \\
\hline & & & & & & & & & & $1.377 \mathrm{E}+05$ & & 1.59 \\
\hline \multirow[t]{2}{*}{55} & CS 137 & 661.66 & 662.52 & 1 & 0 & & $6.502 \mathrm{E}+04+$ & 1.33 & & & & \\
\hline & & & & & & & & & & $6.502 \mathrm{E}+04$ & & 1.33 \\
\hline \multirow[t]{5}{*}{63} & EU 154 & 756.81 & 757.58 & 2 & 512 & 40 ZR 95 & $9.715 \mathrm{E}+05+$ & 1.52 & & & & \\
\hline & & 996.33 & 997.11 & 2 & 0 & & $2.373 E+03+$ & 7.12 & & & & \\
\hline & & 1004.77 & 1005.35 & 2 & 512 & & $2.433 E+03+$ & 4.41 & & & & \\
\hline & & 1274.54 & 1275.00 & 1 & 0 & $11 \mathrm{NA} 22$ & $1.938 \mathrm{E}+03+$ & 3.51 & & & & \\
\hline & & & & & & & & & & $1.970 E+03$ & & 5.82 \\
\hline
\end{tabular}

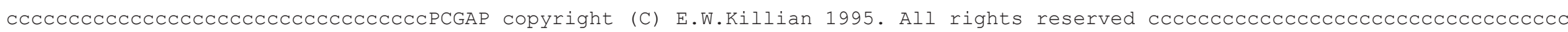


Rev. 4

Analysis of Individual Compact Fission Product Inventory and Burnup for the AGR-1 TRISO Experiment using

Title: Gamma Spectrometry

ECAR No:: 1682

ECAR Rev. No.: 3

Project File No.: 23843

Date: 05/21/2014

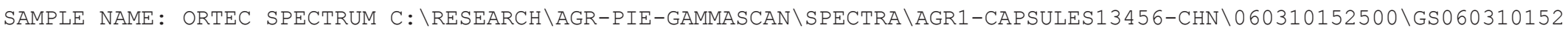

SAMPLE ID:

SAMPLE COLLECTION (DATE/TIME): $60310 / 2331$

SAMPLE COUNTED (DATE/TIME) : $\quad 60310 / 2331$

SAMPLE QUANTITY: $1.00000 \mathrm{E}+00$ QUANTITY UNITS: SMPL

\begin{tabular}{|c|c|c|c|c|c|c|c|}
\hline RADIONUC & CLIDE & SAMPLE & ACTIVITY (uCi/SMPL) & $\div$ ERROR (1-SIGMA) & SAMPLE & ACTIVITY +- & STD DEV \\
\hline $11 \mathrm{NA}$ & 22 & & $6.881 \mathrm{E}+02$ & 3.51 & & $(6.9+1-$ & $0.2) E+02$ \\
\hline $18 \mathrm{AR}$ & 41 & & $7.393 E+01$ & 15.67 & & $7.4+1-$ & $1.2) \mathrm{E}+01$ \\
\hline $19 \mathrm{~K}$ & 40 & & $3.568 \mathrm{E}+02$ & 24.85 & & $(3.6+1-$ & $0.9) \mathrm{E}+02$ \\
\hline $29 \mathrm{CU}$ & 66 & & $2.314 \mathrm{E}+04$ & 3.33 & & $(2.31+/-$ & $0.08) E+04$ \\
\hline $32 \mathrm{GE}$ & 75 & & $2.634 E+02$ & 76.86 & & $(3 .+1-$ & 2.) $\mathrm{E}+02$ \\
\hline $34 \mathrm{SE}$ & 75 & & $4.744 \mathrm{E}+01$ & 76.86 & & $5 .+1-$ & 4.) $E+01$ \\
\hline $39 \mathrm{Y}$ & 91 & & $2.230 \mathrm{E}+04$ & 24.46 & & $(2.2+1-$ & $0.6) \mathrm{E}+04$ \\
\hline $40 \mathrm{ZR}$ & 95 & & $7.804 \mathrm{E}+04$ & 1.23 & & $(7.80+/-$ & $0.10) E+04$ \\
\hline $41 \mathrm{NB}$ & 95 & & $1.568 \mathrm{E}+05$ & 1.61 & & $(1.57+/-$ & $0.03) E+05$ \\
\hline $44 \mathrm{RU}$ & 103 & & $1.617 \mathrm{E}+04$ & 0.76 & & $(1.62+/-$ & $0.01) \mathrm{E}+04$ \\
\hline $44 \mathrm{RU}$ & 105 & & $7.635 \mathrm{E}+04$ & 1.48 & & $(7.63+/-$ & $0.11) \mathrm{E}+04$ \\
\hline 45RURF & $\mathrm{H} 106$ & & $1.377 \mathrm{E}+05$ & 1.59 & & $(1.38+/-$ & $0.02) E+05$ \\
\hline
\end{tabular}


Analysis of Individual Compact Fission Product Inventory and Burnup for the AGR-1 TRISO Experiment using

Title: Gamma Spectrometry

ECAR No.: 1682

ECAR Rev. No.: 3

Project File No.: 23843

Date: $05 / 21 / 2014$

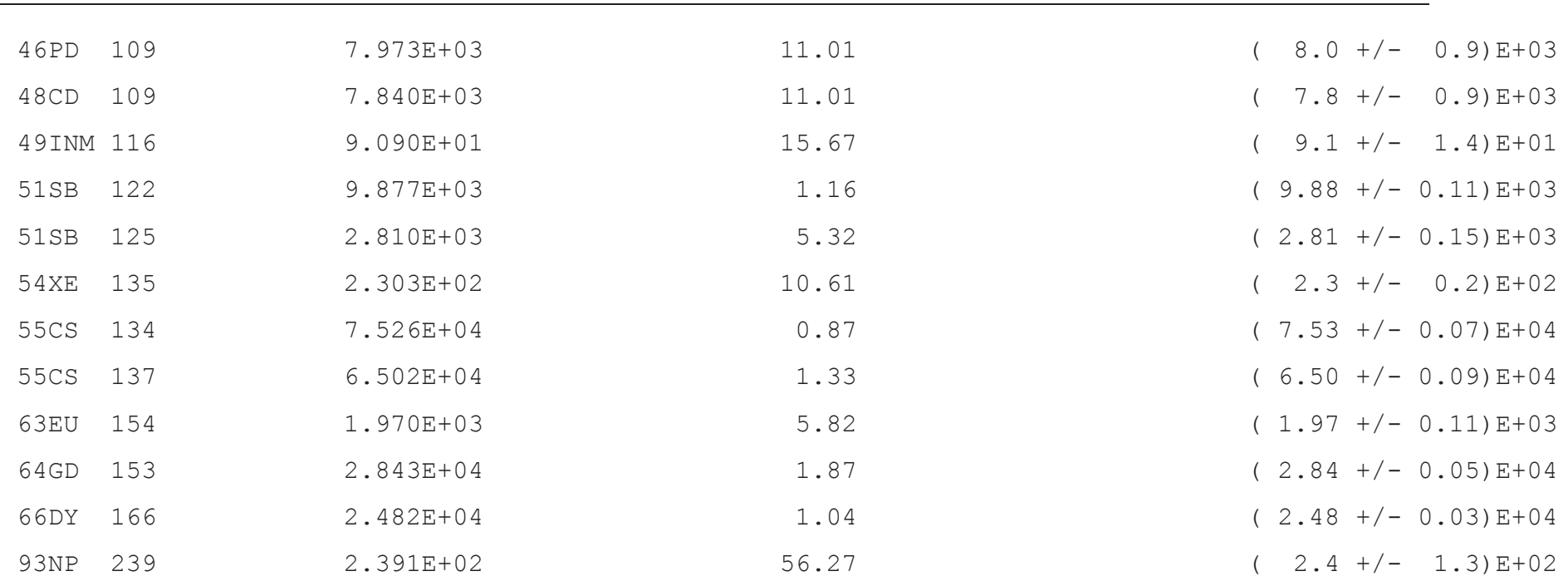


Analysis of Individual Compact Fission Product Inventory and Burnup for the AGR-1 TRISO Experiment using Title: Gamma Spectrometry

\subsection{Appendix B - Listing File Activity Stripping Script and Output}

The Listing file is a detailed document that contains a great deal of information that is not necessarily needed to analyzed the fission product inventory of the compacts. The important information is the activity of a specific isotope and its uncertainty. A python script was written to strip the activities of the isotopes of interest from the .LIS files. This script would also look beyond the positively identified isotopes by PC-GAP to try to identify the activity of other isotopes by the presence or absence of their primary characteristic peak. This feature was primarily used to find the activity of Ag-110m if its primary peak at $657 \mathrm{keV}$ was obscured by a large Cs-137 peak at $662 \mathrm{keV}$. This feature could also be used if an isotope's primary peak had an energy shift that was slightly outside of the PC-GAP allowable energy difference between the measured peak and the library energy. PC-GAP has a delta E difference of $1.0 \mathrm{keV}$, while this script will find peaks in the delta E range of $2.0 \mathrm{keV}$.

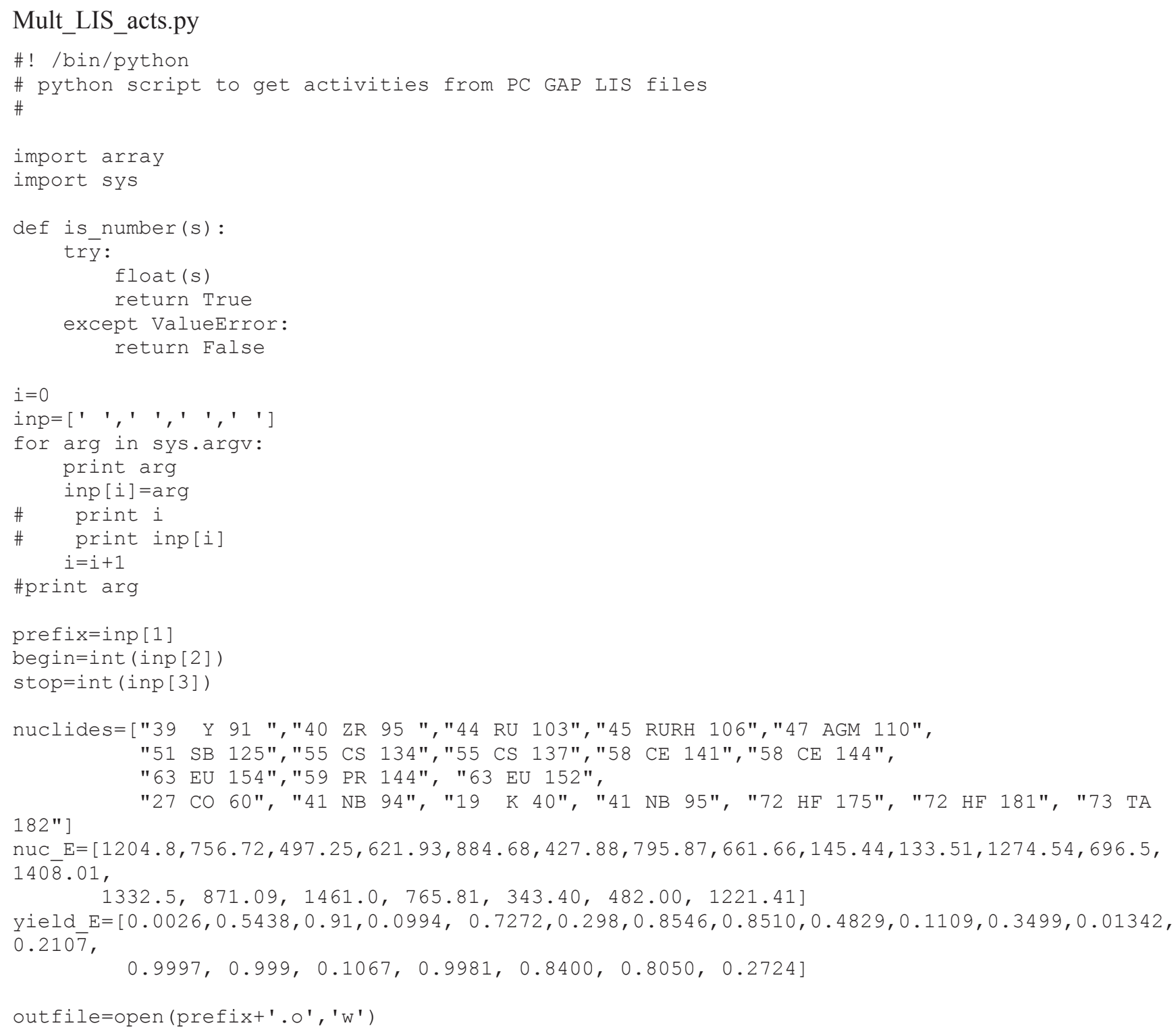


Analysis of Individual Compact Fission Product Inventory and Burnup for the AGR-1 TRISO Experiment using Title: Gamma Spectrometry

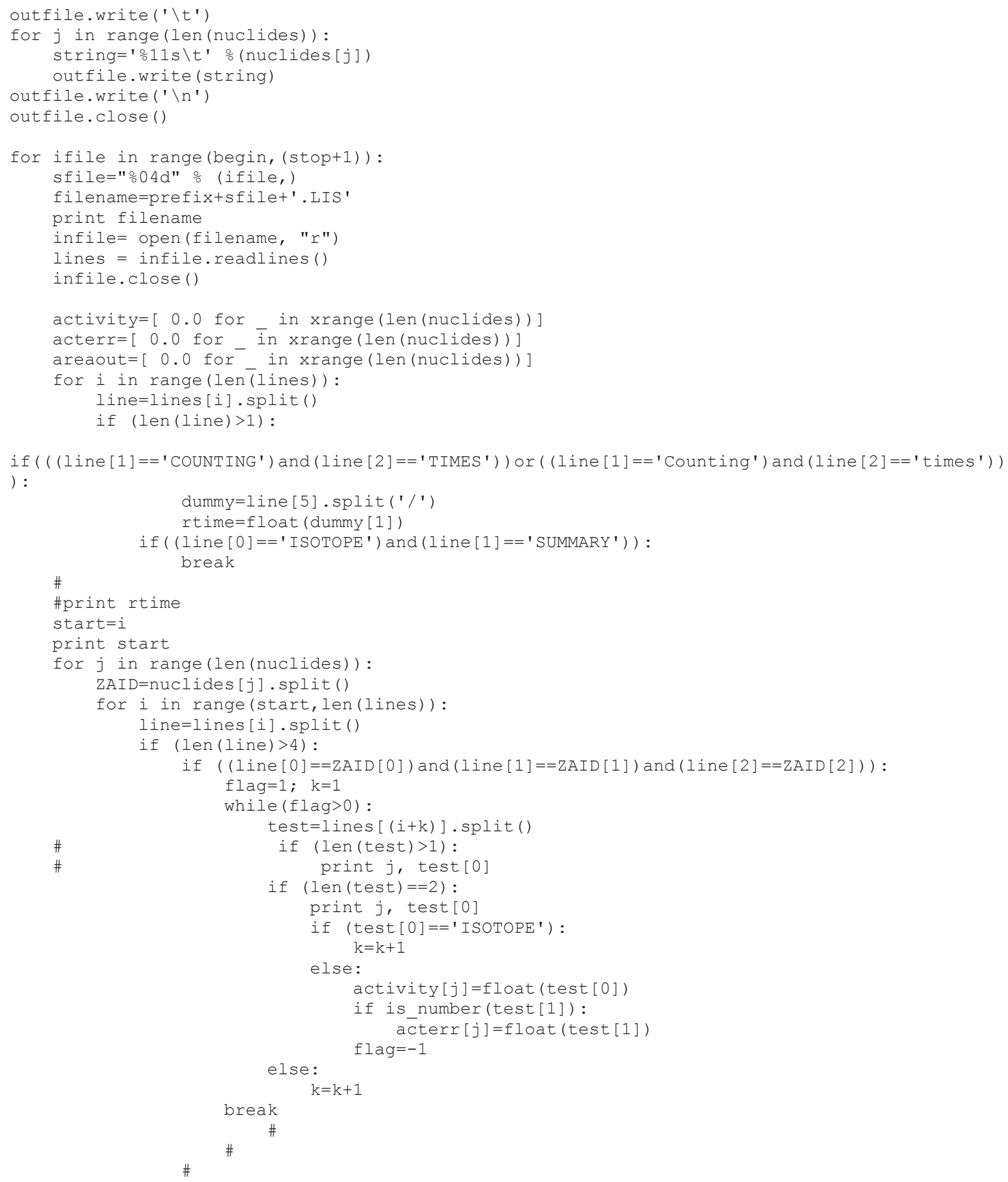


Analysis of Individual Compact Fission Product Inventory and Burnup for the AGR-1 TRISO Experiment using Title: Gamma Spectrometry

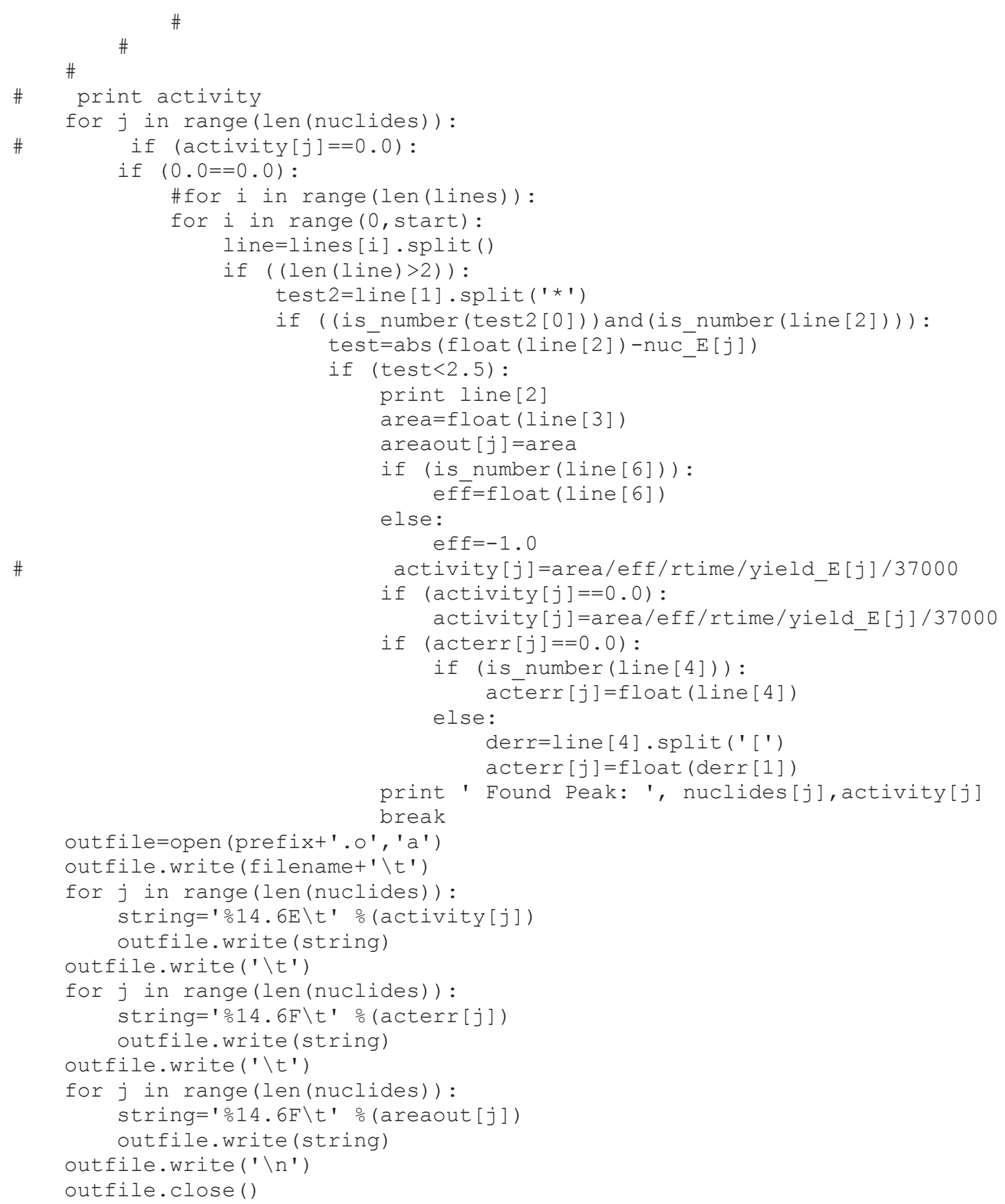

OUTPUT of Mult LIS acts.py

The script is invoked at the command line using the following command format:

: > Mult_LIS_acts.py <file prefix> <start number> <end number>

The files analyzed in this work were saved with names that had the following form: $<$ file_prefix $><$ Number_in_series $>$.LIS. The $<$ file prefix $>$ identified which set of PGS scans the file was a part of. The script will analyze files named $<$ file 
Rev. 4

Analysis of Individual Compact Fission Product Inventory and Burnup for the AGR-1 TRISO Experiment using

Title: Gamma Spectrometry

ECAR No.: 1682

ECAR Rev. No.: 3

Project File No.: 23843

Date: $05 / 21 / 2014$

prefix $><$ start number $>$.LIS to $<$ file prefix $><$ end number $>$.LIS. The included output is for a single file analyzed by the script. If multiple files are analyzed their results are output on separate lines of the output file. Although the output appears on several lines in this document, the resulting text file contains only 1 line per analyzed file. The output file for the script is named $<$ file prefix $>$.o.

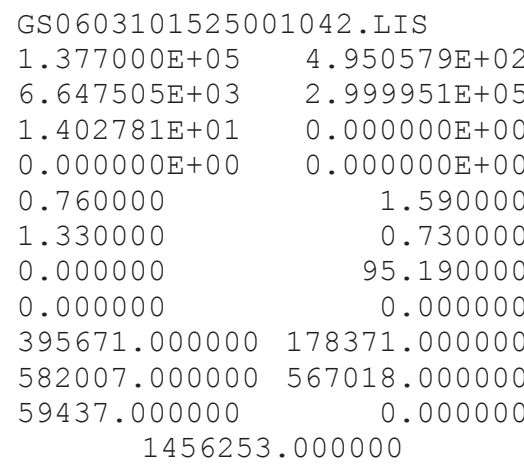

2

$\begin{array}{rcr}.230000 \mathrm{E}+04 & 7.804000 \mathrm{E}+04 & 1.617000 \mathrm{E}+04 \\ 2.810000 \mathrm{E}+03 & 7.526000 \mathrm{E}+04 & 6.502000 \mathrm{E}+04 \\ 1.970000 \mathrm{E}+03 & 4.477197 \mathrm{E}+05 & 0.000000 \mathrm{E}+00 \\ 3.568000 \mathrm{E}+02 & 1.568000 \mathrm{E}+05 & 0.000000 \mathrm{E}+00 \\ 24.460000 & 1.230000 & \\ 4.990000 & 5.320000 & 0.870000 \\ 0.430000 & 5.820000 & 1.740000 \\ 0.000000 & 24.850000 & 1.610000 \\ 0.000000 & 464.000000 & \\ 144835.000000 & 3049.000000 & 11378.000000 \\ 56897.000000 & 586434.000000 & 4597.000000 \\ 91.000000 & 0.000000 & 233.000000 \\ 0.000000 & 0.000000 & 0.000000\end{array}$

